Preprint typeset in JHEP style - PAPER VERSION

\title{
Coupled dark matter-dark energy in light of near universe observations
}

\author{
Laura Lopez Honorez ${ }^{1}$, Beth A. Reid ${ }^{2}$, Olga Mena ${ }^{3}$, Licia Verde ${ }^{4,2}$ and Raul \\ Jimenez $z^{4,2}$. \\ ${ }^{1}$ Physics Department and Instituto de Fisica Teorica UAM/CSIC, 28049 Cantoblanco, \\ Madrid, Spain and \\ Service de Physique Théorique, ULB, 1050 Brussels, Belgium \\ ${ }^{2}$ Institute for Sciences of the Cosmos (ICC), University of Barcelona and IEEC, \\ Barcelona 08028, Spain \\ ${ }^{3}$ Instituto de Fisica Corpuscular, IFIC, CSIC and Universidad de Valencia, Spain \\ ${ }^{4}$ ICREA (Institucio Catalana de Recerca i Estudis Avançats)
}

ABSTRACT: Cosmological analysis based on currently available observations are unable to rule out a sizeable coupling among the dark energy and dark matter fluids. We explore a variety of coupled dark matter-dark energy models, which satisfy cosmic microwave background constraints, in light of low redshift and near universe observations. We illustrate the phenomenology of different classes of dark coupling models, paying particular attention in distinguishing between effects that appear only on the expansion history and those that appear in the growth of structure. We find that while a broad class of dark coupling models are effectively models where general relativity (GR) is modified -and thus can be probed by a combination of tests for the expansion history and the growth of structure-, there is a class of dark coupling models where gravity is still GR, but the growth of perturbations is, in principle modified. While this effect is small in the specific models we have considered, one should bear in mind that an inconsistency between reconstructed expansion history and growth may not uniquely indicate deviations from GR. Our low redshift constraints arise from cosmic velocities, redshift space distortions and dark matter abundance in galaxy voids. We find that current data constrain the dimensionless coupling to be $|\xi|<0.2$, but prospects from forthcoming data are for a significant improvement. Future, precise measurements of the Hubble constant, combined with high-precision constraints on the growth of structure, could provide the key to rule out dark coupling models which survive other tests. We shall exploit as well weak equivalence principle violation arguments, which have the potential to highly disfavour a broad family of coupled models. 


\section{Contents}

1. Introduction 1

2. Two times two families of dark coupling models 3

2.1 Dark coupling models and modified gravity 3

2.2 Background evolution 5

2.3 Linear perturbations $\quad 6$

2.4 Instabilities 8

3. Distinguishing dark coupling models from arbitrary uncoupled $w(z)$ dark $\begin{array}{ll}\text { energy models } & 10\end{array}$

4. Low Redshift Observational Probes of Dark Coupling 13

4.1 Background High-z vs Low-z quantities mis-match: a worked example with Hubble constant measurements 14

$\begin{array}{lll}4.2 & \text { Effects on the skewness } & 16\end{array}$

$\begin{array}{lll}4.3 & \text { Peculiar Velocities } & 17\end{array}$

4.3.1 Constraints from peculiar velocities: Local bulk flows 18

4.3.2 Constraints from peculiar velocities: redshift space distortions 21

4.4 Weak equivalence principle violation (WEPV) constraints 23

4.4.1 Coupled scalar field as an example of DEvel $Q \propto \rho_{\mathrm{dm}}$ models $\quad 23$

4.4.2 DEvel $Q \propto \rho_{\text {de }}$ model $\quad 25$

4.5 Constraints from matter abundance in galaxy voids 26

5. Conclusions 28

A. Background evolution in models with $Q=\xi \mathcal{H} \rho_{\mathrm{de}, \mathrm{dm}} \quad 30$

B. Cosmological parameters according to WMAP 5 constraint 31

\section{Introduction}

Cosmological probes [1-7] indicate that the universe we observe today possesses a flat geometry and a mass energy density made of $\sim 30 \%$ baryonic plus cold dark matter and $70 \%$ dark energy, responsible for the late-time accelerated expansion. The most economical description of the cosmological measurements attributes the dark energy to a Cosmological Constant (CC) in Einstein's equations, representing an invariable vacuum energy density, with constant equation of state $w=-1$. However, from the quantum field theory approach, the predicted energy for the vacuum fluctuations is $\sim 120$ orders of magnitude larger than 
the observed value. This situation is the so-called CC problem. In addition, there is no proposal which explains naturally why the matter and the vacuum energy densities give similar contributions to the universe's energy budget at this moment in the cosmic history. This is the so-called why now? problem, and a possible way to alleviate it is to assume a time varying, dynamical fluid. The quintessence option consists of a cosmic scalar quintessence field which changes with time and varies across space, and it is slowly approaching its ground state e.g., [8-13]. The quintessence equation of state is generally not constant through cosmic time. In principle, the quintessence field may couple to other fields. In practice, observations strongly constrain the couplings to ordinary matter [14]. In addition, due to the the dynamical nature of the quintessence field, any coupling to the baryons would lead to time variation of the constants of nature, which are being tightly constrained see e.g., [15] and references therein. However, interactions within the dark sectors, i.e. between dark matter and dark energy, are still allowed by observations. A non-zero coupling in the dark sector could affect significantly the expansion history of the universe and the density perturbation evolution, changing the growth history of cosmological structures, see Refs. [16-28]. A number of studies have been devoted to analyze the constraints from Cosmic Microwave Background (CMB), large scale structure (LSS), Supernovae Ia and Baryon Acoustic oscillations (BAO) on a variety of interacting models [24,26,28-35]. Forecasts from CMB experiments, as the on going Planck ${ }^{1}$ and future satellite missions e.g., [36] on coupled cosmologies have been recently addressed [37].

Coupled cosmologies, in order to satisfy CMB constraints, predict values for the cosmological parameters today which may differ substantially from the parameters values within non-interacting cosmologies. In order to fit high-precision CMB data available today, coupled cosmologies can hide their effects at very low redshifts. Therefore, low redshift probes are highly complementary and thus powerful to constrain interacting dark sector models. In this paper we focus on near-universe, low-redshift constraints in a variety of coupled dark matter-dark energy models. We explore the phenomenology of coupled models and consider what type of low-redshift observations are most suitable to improve present constraints. We pay attention in distinguishing between effects that appear only on the expansion history and that can thus be tested with observations such as BAO and Supernova Ia and those that appear in the growth of structure. In the spirit of Ref. [38] we shall exploit galaxy velocities and weak equivalence principle violation arguments as additional probes to tighten interactions among the dark sectors. We present as well unexplored, powerful constraints arising from dark matter abundance in voids, as well as from future, precise measurements of the Hubble constant $H_{0}$. Throughout this paper we assume a flat spatial geometry.

It was suggested that the dynamical equilibrium of collapsed structures such as galaxy cluster could advocate in favour of an interaction between dark matter and dark energy [39]. The idea is that the virial theorem is modified by the energy exchange between the dark sectors leading to a bias in the estimation of the virial masses of clusters when the usual virial conditions are employed. This provides an additional near universe probe of the dark

\footnotetext{
${ }^{1}$ http://www.sciops.esa.int/index.php?project=PLANCK
} 
coupling. By comparing weak lensing and X-ray mass-observables to the virial masses, the authors of $[40,41]$ claim that available data suggest that a small coupling could be present involving the decay of dark energy into dark matter. Notice that they also warn the reader that the lack of knowledge about the errors arising from systematics could weaken their conclusions.

In Section 2 we propose a classification of interacting cosmologies into two broad families (DEvel and DMvel) and, in each of these families, two classes, depending on the scaling of the coupling with the matter or dark energy densities. This section presents also the background and the linear perturbation evolution for the different cases and discuss instabilities. Section 3 focuses on the phenomenology of these models compared to a $\Lambda \mathrm{CDM}$ model and to uncoupled models with arbitrary equation of state parameter for dark energy. Section 4 summarizes current constraints for interacting models and future prospects, devoting separate subsections to near universe $H_{0}$ measurements, skewness tests, dark matter velocities and void dark matter abundances. Finally, we draw our conclusions in Section 5.

\section{Two times two families of dark coupling models}

In this section we present the background and linear perturbation theory results for dark coupling models in general and then in the context of specific forms of the coupling. The models that we consider are naturally divided by two features of the model: whether the assumed momentum transfer is zero in the dark energy rest frame versus the dark matter rest frame. In each of these cases the the energy transfer could be taken to be proportional to $\rho_{\mathrm{de}}$ or to $\rho_{\mathrm{dm}}$.

\subsection{Dark coupling models and modified gravity}

At the level of the stress-energy tensor it is always possible to introduce an interaction between the fluids of the dark sector in the following way [42]:

$$
\nabla_{\mu} T_{(\mathrm{dm}) \nu}^{\mu}=Q_{\nu} \quad \text { and } \quad \nabla_{\mu} T_{(\mathrm{de}) \nu}^{\mu}=-Q_{\nu}
$$

The 4-vector $Q_{\nu}$ governs the energy-momentum transfer between the dark components and $T_{(\mathrm{dm}) \nu}^{\mu}$ and $T_{(\mathrm{de}) \nu}^{\mu}$ are the energy-momentum tensors for the dark matter and dark energy fluids, respectively. Different expressions for the form of $Q_{\nu}$, that arise from a variety of motivations, can be found in the literature. Here we attempt to classify the couplings in broad families, based in the different phenomenology -from the astrophysical and cosmological point of view- they display.

We consider two families of four momentum-energy transfer $Q_{\nu}$. In the first family of models (DEvel), the momentum exchange $Q_{\nu}$ is parallel to the dark energy four velocity ${ }^{2}$ $u_{\nu}^{(\mathrm{de})}$ :

$$
Q_{\nu}=Q u_{\nu}^{(\mathrm{de})} / a \quad(\mathrm{DEvel})
$$

\footnotetext{
${ }^{2}$ The scale factor in the denominator is introduced because -at least at linear order- the velocity $u$ is $\propto a$, see Eq. (2.11).
} 
In the second family of models (DMvel), $Q_{\nu}$ is parallel to the dark matter four velocity $[21-24,26,27,38] u_{\nu}^{(\mathrm{dm})}$ :

$$
Q_{\nu}=Q u_{\nu}^{(\mathrm{dm})} / a \quad(\mathrm{DMvel})
$$

DEvel models $\propto \rho_{\mathrm{dm}} u_{\nu}^{(\mathrm{de})}$ include all quintessence coupled models, see for instance, Refs. [10, 16, 23, 32, 38, 43-51]. In DEvel models, there is no momentum transfer to the dark energy frame, so that momentum must be conserved in the dark matter frame. This implies a fractional increase in the dark matter peculiar velocity equal and opposite to the fractional change in energy density due to the presence of a coupling. This effect can be interpreted as an extra source of acceleration for the dark matter fluid, that will appear clearly in the dark matter velocity perturbation equation, see $\S 3$. By contrast, in DMvel models both momentum and energy density are transferred from the dark matter system to the dark energy one, and therefore the dark matter peculiar velocity field does not have this apparent force.

The extra force effect in DEvel models should not come as a surprise: many quintessence coupled field models that appear in the literature can be written as a scalar-tensor BransDicke [52] gravity theory. Let us also recall that $f(R)$ gravity theories correspond to generalized Brans Dicke (BD) theory with a $\mathrm{BD}$ parameter $w_{\mathrm{BD}}=0$ or $=-3 / 2$, see e.g. [53] and references therein. The equations in the Einstein frame contain a new scalar field which satisfies Eqs. (2.1), being the energy momentum exchange $Q_{\nu}$ proportional to its 4 -velocity (see e.g. [54]).

The assumption of such a scalar interaction (DEvel) in the dark sector makes the acceleration of visible and dark matter particles different, inducing a "fifth force" effect (only for the dark matter), that is, a violation of the equivalence principle, for which the Eötvös experiments constraints do not apply. As noted by Ref. [55], a large-scale fifth force in the dark sector might have substantial effect as a mis-match in the relative distribution of baryons and dark matter. DEvel models are therefore effectively "modified gravity" models. It is well known that deviations from the simpler $\Lambda \mathrm{CDM}$ paradigm in the form of general relativity (GR) modifications can be constrained following two approaches: a) at the background evolution level as inconsistencies between the high-redshift and the lowredshift universe $[56,57]$ and $b$ ) at the growth of perturbation level: a modified gravity model with the same expansion history as $\Lambda \mathrm{CDM}$ model, has a different growth of the dark matter structures. This has been extensively discussed in Refs. [58-60]. In fact, for some specific cases, there is a third possible approach: $c$ ) using weak equivalence principle violation (WEPV) constraints.

In the following, we will see that option a) also applies to DMvel models and we illustrate its potential in the context of those models in $\S 4.1$. This approach only probes the background evolution and thus cannot be used alone to distinguish modified gravity or dark coupling from a minimally coupled dark energy model with general, time-dependent equation of state parameter $w(z)$. For what concerns approach $b$ ), we show in $\S 3$ that the growth of perturbations in DMvel and DEvel coupled models can differ from the growth in an uncoupled model with identical background history. In the case of DEvel models (which in practice are effectively modified gravity models) it is already very well known 
that growth can provide the key to break the existing degeneracy at the background level among GR and modified gravity. However, for DMvel models, the result we obtain here is a counterexample to what is commonly accepted in the literature: DMvel models are not a modification of gravity, but their growth can in principle still differ from the growth in a GR dynamical dark energy model which possesses the same background history since $\rho_{\mathrm{dm}}(z)$ does not behave like dust (i.e., $\left.\propto(1+z)^{3}\right)$. See also [61]. Finally, approach $c$ ) is specific of DEvel models (i.e., it is the smoking gun of those models) and we will consider it quantitatively in $\S 4.4$.

\subsection{Background evolution}

In Eq.( 2.2) and ( 2.3), $Q$ drives the energy exchange between dark matter and dark energy. Indeed, one can easily show that for DEvel and DMvel families, the evolution equations for the dark matter and dark energy background energy densities reduce to:

$$
\begin{aligned}
\dot{\rho}_{\mathrm{dm}}+3 \mathcal{H} \rho_{\mathrm{dm}} & =Q, \\
\dot{\rho}_{\mathrm{de}}+3 \mathcal{H} \rho_{\mathrm{de}}(1+w) & =-Q .
\end{aligned}
$$

$\rho_{\mathrm{dm}}\left(\rho_{\mathrm{de}}\right)$ denotes the dark matter (dark energy) energy density, the dot indicates derivative with respect to conformal time $d \tau=d t / a, \mathcal{H}=\dot{a} / a$ and $w=P_{\mathrm{de}} / \rho_{\mathrm{de}}$ is the dark-energy equation of state ( $P$ denotes the pressure). We work with the Friedman-Robertson-Walker metric, assuming a flat universe and pressureless dark matter $w_{\mathrm{dm}}=P_{\mathrm{dm}} / \rho_{\mathrm{dm}}=0$. The sign of $Q$ determines the direction of energy transfer. For positive $Q$, the energy flows from the dark energy system to dark matter one. For negative $Q$, the energy flow is reversed. Note that if $Q<0, \rho_{\mathrm{dm}}$ decreases with time because dark matter is being transformed into dark energy. The presence of a coupling $Q$ also changes the dark matter and dark energy redshift dependence acting as an extra contribution to their effective equation of state. Indeed, the effective background equation of state for the two fluids are $[24,27]$

$$
w_{\mathrm{dm}}^{\mathrm{eff}}=-\frac{Q}{3 \mathcal{H} \rho_{\mathrm{dm}}} ; \quad w_{\mathrm{de}}^{\mathrm{eff}}=w+\frac{Q}{3 \mathcal{H} \rho_{\mathrm{de}}} .
$$

Therefore e.g., a negative $Q$ yields an effective equation of state for dark energy $w_{\mathrm{de}}^{\text {eff }}$ that is more negative than $w \equiv P_{\mathrm{de}} / \rho_{\mathrm{de}}$. A negative $Q$ will also contribute as a positive pressure in the dark matter background equation. Note however that the deceleration parameter satisfies, regardless of the presence of non-zero dark coupling,

$$
q=-\frac{\dot{\mathcal{H}}}{\mathcal{H}^{2}}=\frac{1}{2}\left(1+3 w \Omega_{\mathrm{de}}\right)
$$

where $\Omega_{\text {de }}$ is the time dependent relative dark energy density. Therefore, we would still require $w<-1 / 3$ to have a universe with accelerated expansion.

Because of the unknown nature of the dark sector, to-date there is no prescription in fundamental theory for a physically-motivated model for the coupling between the dark

matter and dark energy fluids. The interaction term $Q$ is currently mostly chosen in a phenomenological way. For the models considered in the literature in which the dark 
coupling depends linearly on the dark sector energy densities, for each of the two families DEvel and DMvel, we propose the definition of two sub-classes of models:

$$
\begin{aligned}
& Q=\Sigma \rho_{\mathrm{dm}} \quad(\text { class I }) \\
& Q=\Sigma \rho_{\mathrm{de}} \quad(\text { class II })
\end{aligned}
$$

where $\Sigma$ denotes the interaction rate. The former classification should be considered as a "basis set" for the study of coupled models. For example, the coupling $Q=\Sigma_{1} \rho_{\mathrm{dm}}+\Sigma_{2} \rho_{\mathrm{de}}$ is also present in the literature $[22,30,33,40,41]$ and it does not strictly belongs to class I or class II. We trust however that our analysis can guide the reader to determine what would be the relevant test to apply depending on the contribution that dominates at the scale or redshift corresponding to the probes under investigation. To gain physical insight on the phenomenology of coupled models we concentrate here on the simple cases of "pure" class I or class II models in both DMvel or DEvel scenarios.

In coupled quintessence models $\Sigma$ is proportional to the time derivative of the quintessence field, see e.g. Refs. $[10,16,23,32,38,43-51]$. More phenomenologically motivated models have taken $\Sigma$ to be proportional to the Hubble expansion rate, since the former cosmological parameter has the appropriate time dependence ${ }^{3}$. Even if the $\Sigma \propto \mathcal{H}$ choice [21-24], is much more easy to handle mathematically than the $\Sigma \propto H_{0}$ case [21,27] (being $H_{0}$ the present-day value of the Hubble parameter), we will consider both possibilities in this paper.

It is important to keep in mind that the expansion history does not depend on the choice DEvel or DMvel (but does depend on the form of $Q$ ). Unfortunately, an analytic form for the expansion history $H(z)$ cannot be written for generic $Q$. The only case where it can be done is in the case of $Q=\xi \mathcal{H} \rho_{\mathrm{de}, \mathrm{dm}}$ (a mathematically easy to handle coupling) where both the coupling $\xi$ and the equation of state parameter $w$ are constant (see Eqs. (A.1) and (A.3) in Appendix A and e.g., [24]). Even if DMvel and DEvel models provide the same background history, the perturbation evolution is dramatically different, as we shall see in the next subsection. Therefore, geometrical probes alone are unable to distinguish among the two of them -or distinguish dark coupling from a uncoupled dark energy model with a $w(z)-$, even if, as we will see in $\S 3$, the two models are fundamentally different and different from minimally coupled dark energy models.

\subsection{Linear perturbations}

In the Newtonian gauge, the perturbed FRW metric at linear order in scalar perturbations is given by:

$$
d s^{2}=a^{2}\left[-(1+2 \Psi) d \tau^{2}+(1-2 \Phi) d x_{i} d x^{i}\right],
$$

where $a$ is the scale factor, $\tau$ is the conformal time, $x^{i}$ is the comoving coordinate, and $\Psi$ and $\Phi$ are the scalar metric perturbations in the Newtonian gauge.

The four velocity of a fluid reads

$$
u_{\nu}=a\left(-(1+\Psi), v_{i}\right),
$$

\footnotetext{
${ }^{3}$ For more discussion on a possible physical interpretation of this choice, see e.g., [21, 22].
} 
where $v_{i}$ is the fluid component peculiar velocity. Since baryons are not coupled to the dark energy fluid, the continuity and the Euler equations for the baryons after decoupling are equivalent to those in uncoupled cosmologies:

$$
\begin{aligned}
& \dot{\delta}_{\mathrm{b}}=-\theta_{\mathrm{b}}+3 \dot{\Phi} ; \\
& \dot{\theta}_{\mathrm{b}}=-\mathcal{H} \theta_{\mathrm{b}}+k^{2} \Psi,
\end{aligned}
$$

where $\delta \equiv \delta \rho / \rho$ is the fluid energy density perturbation and $\theta \equiv \partial_{i} v^{i}$ is the divergence of the fluid proper velocity $v^{i}$. We have assumed that baryons behave as a barotropic fluid with $d P_{b} / d \rho_{b}=\delta P_{b} / \delta \rho_{b}=0$, and we will assume the same properties for dark matter. For the coupled dark matter and dark energy components, we obtain, at linear order:

$$
\begin{aligned}
\dot{\delta}_{\mathrm{dm}}= & -\left(\theta_{\mathrm{dm}}-3 \dot{\Phi}\right)+\frac{Q}{\rho_{\mathrm{dm}}}\left(\frac{\delta Q}{Q}-\delta_{\mathrm{dm}}+\Psi\right) \\
\dot{\theta}_{\mathrm{dm}}= & -\mathcal{H} \theta_{\mathrm{dm}}+(1-b) \frac{Q}{\rho_{\mathrm{dm}}}\left(\theta_{\mathrm{de}}-\theta_{\mathrm{dm}}\right)+k^{2} \Psi \\
\dot{\delta}_{\mathrm{de}}= & -(1+w)\left(\theta_{\mathrm{de}}-3 \dot{\Phi}\right)-\frac{Q}{\rho_{\mathrm{de}}}\left(\frac{\delta Q}{Q}-\delta_{\mathrm{de}}+\Psi\right) \\
& -3 \mathcal{H}\left(\hat{c}_{s \mathrm{de}}^{2}-w\right)\left[\delta_{\mathrm{de}}+\mathcal{H}\left(3(1+w)+\frac{Q}{\rho_{\mathrm{de}}}\right) \frac{\theta_{\mathrm{de}}}{k^{2}}\right] \\
\dot{\theta}_{\mathrm{de}}= & -\mathcal{H}\left(1-3 \hat{c}_{s \mathrm{de}}^{2}-\frac{\hat{c}_{s \mathrm{de}}^{2}+b}{1+w} \frac{Q}{\mathcal{H} \rho_{\mathrm{de}}}\right) \theta_{\mathrm{de}}+\frac{k^{2}}{1+w} \hat{c}_{s \mathrm{de}}^{2} \delta_{\mathrm{de}}+k^{2} \Psi-b \frac{Q}{\rho_{\mathrm{de}}} \frac{\theta_{\mathrm{dm}}}{1+w} .
\end{aligned}
$$

We use the $b$ notation introduced in Ref. [23], where $b=0$ refers to DEvel models with $Q_{\nu} \propto u_{\nu}^{(\mathrm{de})}(2.3)$ and $b=1$ refers to DMvel models with $Q_{\nu} \propto u_{\nu}^{(\mathrm{dm})}(2.2) ; \hat{c}_{s \text { de }}^{2}$ is the dark energy pressure perturbation sound speed in the rest frame of dark energy. For the derivation of Eq. (2.16), we have used the following relation:

$$
\delta P_{\mathrm{de}}=\hat{c}_{s \mathrm{de}}^{2} \delta \rho_{\mathrm{de}}+\left(\hat{c}_{s \mathrm{de}}^{2}-c_{a \mathrm{de}}^{2}\right) \dot{\rho}_{\mathrm{de}} \frac{\theta_{\mathrm{de}}}{k^{2}},
$$

which is valid in both Newtonian and synchronous gauges. If not otherwise stated, in the following, we assume $\hat{c}_{s \text { de }}^{2}=1$ (as for quintessence) ${ }^{4}$. The dark energy density perturbations will not cluster significantly if the sound speed for the dark energy $\hat{c}_{s \text { de }}^{2}=1$, and therefore, in the following, we can safely neglect dark energy perturbations in the perturbation evolution (see also the discussion in Refs. [25,38]).

Notice that the Euler equation for the dark matter fluid is only modified in the first family of models (DEvel) considered here, where $Q_{\nu} \propto u_{\nu}^{(\mathrm{de})}$ case, violating therefore the weak equivalence principle, as we shall see in $\S 4.4$. This feature of DEvel models was previously discussed in $\S 2.1$.

The growth equations for dark matter and baryons can easily be derived from Eqs. (2.12), (2.13), (2.14) and (2.15) going to the Newtonian limit (i.e. for $k \ll \mathcal{H}$ ). The growth equation for dark matter is however rather sensible to the type of coupling.

\footnotetext{
${ }^{4}$ Notice that current data still allows for $c_{s}^{2} \sim 0$. However, in Ref. [62], it is shown that the two limits $\hat{c}_{s}^{2} \sim 0$ and $\hat{c}_{s}^{2} 1$ could be discriminated by future experiments.
} 
The simplest case is the one of DMvel $Q \propto \rho_{\mathrm{dm}}$ (class I) coupled models for which we recover the standard perturbation equations at linear order as well as the growth equation for the two matter fluids $\alpha=\mathrm{dm}$, b:

$$
\delta_{\alpha}^{\prime \prime}=-(2-q) \frac{\delta_{\alpha}^{\prime}}{a}+\frac{3}{2}\left(\Omega_{\mathrm{dm}} \frac{\delta_{\mathrm{dm}}}{a^{2}}+\Omega_{\mathrm{b}} \frac{\delta_{\mathrm{b}}}{a^{2}}\right)
$$

where $q$ is the deceleration parameter of Eq. (2.7) and ${ }^{\prime} \equiv d / d a$. Notice that the evolution equations for baryons are always the standard ones because they are not affected by the coupling introduced in Eq. (2.1). For the dark matter perturbations in DMvel $Q \propto \rho_{\mathrm{dm}}$ models, the difference with non-interacting cosmologies arise exclusively due to the different background evolution of the quantities $\mathcal{H}$ and $\Omega_{\mathrm{dm}}[25,38]$. The growth equation is not modified but the scaling with redshift of $\rho_{\mathrm{dm}}$ is different from that of a conserved pressureless fluid. As a result these models are effectively indistinguishable from minimally coupled dark energy models with a $w(z)$.

For all the other cases, i.e. DMvel $Q \propto \rho_{\text {de }}$ class II and DEvel class I \& II, the dark matter linear perturbation equations and the growth equations are both modified by the presence of a coupling term $Q$. In order to derive the growth equation, an explicit form for the interaction term $Q$ must be assumed, as we shall see in $\S 3$.

\subsection{Instabilities}

The existence of non adiabatic, early time instabilities in coupled models is a well known phenomena $[21-24,27,63,64]$. In brief, the dark coupling term which appears through $\dot{\rho}_{d e}$ in the dark energy pressure perturbations of Eq. (2.18) is a source for early time instabilities at large scales in the dark sector. The latter could arise in coupled models when the dark coupling term dominate on the standard (uncoupled) non adiabatic contributions to the dark energy pressure perturbation. The instability is then rapidly transferred to the others fluids components and to the curvature perturbation related to them, producing a non viable cosmological scenario.

Here we briefly review the instability issue and provide a general recipe to avoid instabilities in the perturbation evolution in the case of a constant equation of state $w$ ( $w_{\mathrm{de}}^{\text {eff }}$ can still vary). This recipe will be relevant throughout the paper, where the range and sign for the dimensionless parameters describing the coupling have been chosen to ensure an instability-free perturbation evolution.

The onset of non-adiabatic instabilities depends on the form of the dark coupling $Q$ (class I or class II), on the dark energy equation of state $w$ and on the $Q_{\nu} 4$-velocity dependence (DMvel or DEvel). We shall define a doom factor $\mathbf{d}$ as [24] which is defined independently of the explicit form of the coupling $Q$ :

$$
\mathbf{d} \equiv \frac{Q}{3 \mathcal{H} \rho_{\mathrm{de}}(1+w)}
$$

If $|\mathbf{d}|>1$, the interaction among the two dark sectors drives the non-adiabatic contribution to the dark energy pressure perturbation. At large scales, or equivalently, at early times 
$(\mathcal{H} / k \gg 1)$, the leading contributions in $Q$, or equivalently in $\mathbf{d}$, to the second order differential equation for $\delta_{\mathrm{de}}$ reads:

$$
\delta_{\mathrm{de}}^{\prime \prime} \simeq 3 \mathbf{d}\left(\hat{c}_{s \mathrm{de}}^{2}+b\right)\left(\frac{\delta_{\mathrm{de}}^{\prime}}{a}+3 b \frac{\delta_{\mathrm{de}}}{a^{2}} \frac{\left(\hat{c}_{s \mathrm{de}}^{2}-w\right)}{\hat{c}_{s \mathrm{de}}^{2}+b}+\frac{3(1+w)}{a^{2}} \delta[\mathbf{d}]\right)+\ldots
$$

where $b=0(b=1)$ applies to DEvel (DMvel) models and ${ }^{\prime} \equiv d / d a$. The sign of the coefficient of $\delta_{\mathrm{de}}^{\prime}$ in Eq. $(2.21)$ is, for $\hat{c}_{s \text { de }}^{2}>0$, equivalent to the sign of the doom factor d. A positive doom factor $\mathbf{d}>1$ can lead to large-scale instabilities. Therefore, a simple recipe to avoid non adiabatic instabilities is to consider as viable models only those in which the energy transfer among the dark sectors $Q$ and the (constant) equation of state of the dark energy component $w$ are such that the doom factor d, Eq. (2.20), is always negative.

Having defined all the tools, we briefly review the instability issue for the coupled models that will be analyzed in the following sections ${ }^{5}$. We have shown that the key parameter is the doom factor, which only depends on background quantities such as $Q$. This means that the stability of the perturbations strongly depends on whether the model is a class I or a class II coupled model. However, the stability of the perturbations shows only a mildly dependence on the DEvel-DMvel classification of the model (see the $b$ dependence of Eq. (2.21)).

For class II models, it is easy to verify that, for $Q=\xi \mathcal{H} \rho_{\mathrm{de}}$ with $\xi$ constant and negative, and $w>-1$, the doom factor is negative. In the following, we shall consider

$$
Q_{\nu}=\xi \mathcal{H} \rho_{\mathrm{de}} u_{\nu}^{\mathrm{dm}} / a \quad \text { and } \quad Q_{\nu}=\xi \mathcal{H} \rho_{\mathrm{de}} u_{\nu}^{\mathrm{de}} / a
$$

with negative dimensionless coupling $\xi$ and $w>-1$. The two models above are, respectively, the DMvel class II and DEvel class II models which we will analyze next.

It was shown in Ref. [24] that for $Q=\xi \mathcal{H} \rho_{\mathrm{dm}}$, the doom factor is always positive assuming $w>-1$. It will also be enhanced by the $\rho_{\mathrm{dm}} / \rho_{\mathrm{de}}$ factor, see Eq. (2.20). A not very attractive solution to solve the problem would be to consider a constant $w<-1$ [22-24]. Another avenue is to use a time dependent equation of state for dark energy. In Ref. [27] it was shown that the DMvel model

$$
Q_{\nu}=-a \Gamma \rho_{\mathrm{dm}} u_{\nu}^{(\mathrm{dm})}, \text { with } w(a)=w_{0} a+w_{e}(1-a)
$$

is stable for a specific range of constant $w_{0}, w_{e}$ and $\Gamma / H_{0}$. This is the DMvel class I model that we shall analyze.

In the case of DEvel class I models, we shall refer to the case of a coupled quintessence model:

$$
Q_{\nu}=\beta \rho_{\mathrm{dm}} \nabla_{\nu} \phi / M_{p}
$$

where $\phi$ is the dark energy field driving the interaction, $M_{p}$ denotes the Plank mass $\left(M_{p}=\right.$ $\left.1 / \sqrt{8 \pi G_{N}}\right)$ and $\beta$ will be considered to be a constant. Note that in this case $u_{\nu}^{(\mathrm{de})} \propto \nabla_{\nu} \phi / \dot{\phi}$.

\footnotetext{
${ }^{5}$ For a detailed treatment of the instability issue in each of the cases reviewed below, we refer the reader to Refs. [21-24,27,63,64]
} 
The early time non adiabatic instabilities discussed here are different from the adiabatic instabilities. These adiabatic instabilities may arise at late times in quintessence coupled models if the sound speed of the total fluid gets negative [65], even if Ref. [64] has shown that the slow-rolling of the quintessence field may avoid such instabilities in some cases. In the following, the term instabilities will refer exclusively to non adiabatic instabilities.

\section{Distinguishing dark coupling models from arbitrary uncoupled $w(z)$ dark energy models}

At the background level, a dynamical, redshift-dependent, effective equation of state $\tilde{w}(z)$, can be mimicked by the combination of a constant equation of state $w$ plus a dark matterdark energy coupling $Q$ [24]. Assume that the true, underlying cosmology possesses an interacting dark energy fluid with constant, non phantom, equation of state $w>-1$. If the background is analyzed setting the coupling to zero, one would reconstruct an effective redshift dependent $\tilde{w}(z)$ :

$$
\tilde{w}(z)=\frac{1}{3} \frac{(1+z) d R_{H} / d z-3 R_{H}}{R_{H}-\tilde{\Omega}_{\mathrm{m}}^{(0)}(1+z)^{3}}
$$

where $R_{H}(z)$ is a function of the background of the coupled model,

$$
R_{H}(z)=\frac{H^{2}(z)}{H_{0}^{2}}
$$

and $\tilde{\Omega}_{\mathrm{m}}^{(0)}$ is matter energy density (dark matter plus baryons) as estimated from the CMB and extrapolated to $z=0$ assuming conserved pressureless dust. An analogous relation between the redshift-dependent equation of state and the luminosity distance and its derivative was firstly presented in Ref. [66].

In the following, we study uncoupled models with $\tilde{w}(z)$ versus interacting DMvel and DEvel class II cosmologies with $Q=\xi \mathcal{H} \rho_{\text {de }}$. We investigate how to distinguish among them, even if the background evolution in these cosmologies is identical. For this particular type of coupling, $H(z)$ is given by Eq. (A.1), where we consider $\xi<0$ to avoid early-time instabilities.

In the class II DMvel and DEvel models we consider here, the energy-momentum transfer is completely negligible at the high redshift relevant to the CMB. Assuming a flat universe and perfect measurements of $\Omega_{\mathrm{dm}} h^{2}, \Omega_{\mathrm{b}} h^{2}$, and the angular diameter distance to the last scattering surface from CMB observations, the amplitude of $\xi$ is degenerate with the physical energy density in dark matter today ${ }^{6}$. Figure I illustrates the values of $\Omega_{\mathrm{dm}}^{(0)} h^{2}$ necessary to fit WMAP 5 year angular diameter distance data, i.e. the first acoustic peak position [1], as a function of the dimensionless coupling constant $\xi$, taking into account that at the redshift of recombination $z_{*}, \Omega_{\mathrm{dm}}\left(z_{*}\right) \simeq\left(\Omega_{\mathrm{dm}}^{(0)}+\Omega_{\mathrm{de}}^{(0)} \xi /\left(3 w_{\text {eff }}\right)\right)\left(1+z_{*}\right)^{3}$, or equivalently, that the dark matter do not evolve as $\Omega_{\mathrm{dm}}^{(0)}(1+z)^{3}$ in the coupled models under study. The values of $\Omega_{\text {de }}$ and $H$ are defined coherently with the flat universe assumption.

\footnotetext{
${ }^{6}$ There is a corresponding degeneracy with $H_{0}$ that will be further quantified in $\S 4.1$.
} 


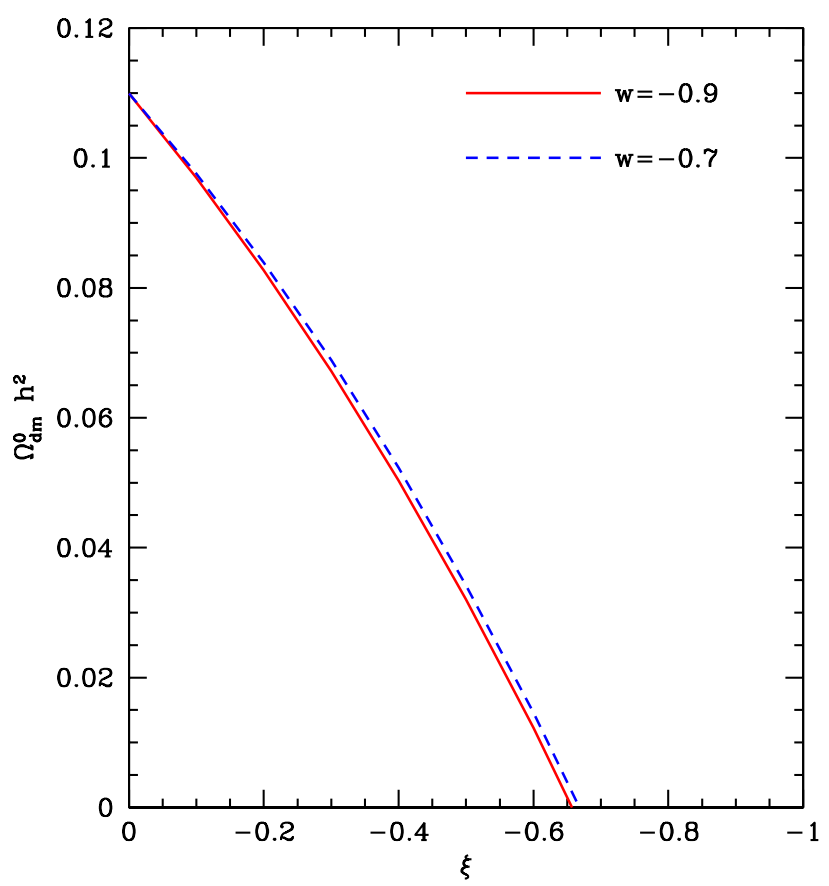

Figure I: Present-day value of the dark matter energy density of the universe for the class II DMvel and DEvel models characterized by $Q=\xi \mathcal{H} \rho_{\mathrm{de}}$ as a function of the parameter $\xi$, necessary to fit WMAP 5 year angular diameter distance data [1] and the physical dark matter and baryon densities at decoupling. The red solid (blue dashed) curve assumes an equation of state of the dark energy component $w=-0.9(w=-0.7)$.

The values of the cosmological parameters $\Omega_{\mathrm{dm}}^{(0)}$ and $H_{0}$, obtained as a function of the coupling $\xi$, are presented in a Table in the Appendix B. The degeneracy direction depends only slightly on the (constant) value of $w$ as shown in Fig. I. In the following, we shall restrict ourselves to $\xi>-0.6$, to ensure positive matter density at $z=0$.

It may be puzzling that dark matter could become negative, however, restricting ourselves to the $\xi$ range above, this would happen in the future and by no means we can avoid it (the couplings $\xi<-0.6$ are already ruled out by a combined analysis of several cosmological data $[24,67])$. An even more troubling feature is that in underdense regions $\rho_{\mathrm{dm}}(x)$ may become negative well before $\Omega_{d m}$ does it globally. This statement depends on the smoothing scale employed to define $\rho_{\mathrm{dm}}(x)$. Therefore, it may happen that at some special point in the Universe, for any value of the coupling, at some point in the past the current description makes the local dark matter density negative. However, notice that this would happen when fluctuations become highly non-linear, a regime which can only be explored with N-body simulations. Rather than an inconsistency of the model this indicates that the model should be seen as an effective, coarse grained description of some more complex and fundamental model.

At the level of perturbations, the phenomenology in these coupled models is simplified in the case where $c_{\mathrm{de}}^{2}$ is large, where dark energy perturbations would be negligible. 
Moreover we will assume in this paper that the perturbations in the expansion rate $\delta H$ can be neglected, see Ref. [28] for more details. In this situation, the rate of energy transfer between dark matter and dark energy is nearly homogeneous. For an energy density transfer, for instance, $\alpha \rho_{\mathrm{dm}}$, the product $\rho_{\mathrm{dm}} \delta_{\mathrm{dm}}$ remains constant, while $\delta_{\mathrm{dm}} \rightarrow \delta_{\mathrm{dm}} /(1-\alpha)$. Therefore we expect very small changes to the lensing signal, $\propto \Omega_{\mathrm{dm}} \delta_{\mathrm{dm}}$. However, since $\delta_{\mathrm{dm}}$ grows, we do expect some enhancement to the growth of structure in these models.

In a more quantitative approach, one can derive the growth equation for dark matter in the Newtonian limit. We obtain:

$$
\delta_{\mathrm{dm}}^{\prime \prime}=-B \frac{\delta_{\mathrm{dm}}^{\prime}}{a}+\frac{3}{2}\left(A \Omega_{\mathrm{dm}} \frac{\delta_{\mathrm{dm}}}{a^{2}}+\Omega_{\mathrm{b}} \frac{\delta_{\mathrm{b}}}{a^{2}}\right),
$$

where

$$
\begin{aligned}
& B=2-q+(2-b) \xi \frac{\rho_{\mathrm{de}}}{\rho_{\mathrm{dm}}}, \\
& A=1+\frac{2}{3} \frac{1}{\Omega_{\mathrm{dm}}} \frac{\rho_{\mathrm{de}}}{\rho_{\mathrm{dm}}}\left[-\xi(1-q-3 w)+\xi^{2}\left(\frac{\rho_{\mathrm{de}}}{\rho_{\mathrm{dm}}}+1\right)\right] .
\end{aligned}
$$

Recall that $b=0$ for DEvel models and $b=1$ for DMvel models. For negative coupling $\xi$, the Hubble friction term $B$ is suppressed and the $A$ contribution to the source term in enhanced. This implies that the dark matter growth in coupled models satisfying Eqs. (2.22) will be larger than in uncoupled models. More generally, this feature is valid for any coupled model in which $Q$ is directly proportional to the dark energy density and $Q / \rho_{\text {de }}$ is negative, see e.g. Ref. [25].

One could be tempted to interpret the change in the source term of Eq. (3.5) as the result of a fifth force for dark matter, the total attractive force between dark matter particles being driven by some effective gravitational constant $G_{\text {eff }}$ with $G_{\text {eff }} / G=A$. This interpretation would mean the violation of the weak equivalence principle. However, such an interpretation is incorrect in the case of $Q_{\nu} \propto u_{\nu}^{(\mathrm{dm})}$, as the dark matter Euler Eq. (2.15) is not modified by the presence of the dark coupling and the velocities for dark matter and baryons are identical.

We analyze now the linear growth in the three possible cosmologies that lie along the one-dimensional degeneracy defined by Fig. I. These three possible cosmologies, which have identical background histories, are: a) an uncoupled, albeit dynamical dark energy cosmology, with a varying equation of state $\tilde{w}(z)$ given by Eq. (3.1), with $H(z)$ given by Eq. (A.1) and assuming that $\Omega_{\mathrm{dm}}$ in Eq. (3.3) scales with $\left.a^{-3}, b\right)$ the coupled $Q \propto \rho_{\mathrm{de}}$ DMvel model with constant $w=-0.9$ given by the first equation of Eqs. $(2.22)$ and $c$ ) the coupled class II $Q \propto \rho_{\text {de }}$ DEvel model with constant $w=-0.9$ given by the second expression of Eqs. (2.22). While in the DMvel model the baryon densities and velocities nearly trace those of the dark matter, this is not the case for the DEvel model. This difference will be carefully explored in $\S 4.3-4.4$. We consider observables probed by lensing $\left(\propto \Omega_{\mathrm{dm}} \delta_{\mathrm{dm}}\right.$, if one ignores geometrical factors) and linear peculiar velocities $\left(\propto \theta_{\mathrm{dm}}\right)$ see also $\S 4$.

Figure II shows the ratio of the values of $\Omega_{\mathrm{dm}} \delta_{\mathrm{dm}}$ and $\theta_{\mathrm{dm}}$ in cases $b$ ) and $c$ ) to their values in an uncoupled model with the same expansion history dictated by the relation in 
Fig. I, for six possible values of the coupling $\xi$. Let us emphasize that in Figure II, $\mathrm{t}$ he quantity $\Omega_{\mathrm{dm}} \delta_{\mathrm{dm}}$ is derived using the solution for $\Omega_{\mathrm{dm}}$ of Eq. (2.4) with non zero coupling, and $\delta_{\mathrm{dm}}$ satisfying the growth equation (3.3). At the background level, the cosmological parameters were chosen to match the angular diameter distance to last scattering surface from CMB observations. $\Omega_{\mathrm{dm}, \mathrm{NC}} \delta_{\mathrm{dm}, \mathrm{NC}}$ refers to the uncoupled model (Not Coupled) with the same expansion history than the coupled model. In the NC model $H(z)$ is constructed assuming that $\Omega_{\mathrm{dm}, \mathrm{NC}}$ has the usual $a^{-3}$ dependence and the dark energy fluid is described by the effective equation of state of Eq. (3.1). The NC matter overdensity field $\delta_{\mathrm{dm}, \mathrm{NC}}$ satisfies equation (2.19). An equivalent approach has been followed to derive $\theta_{\mathrm{dm}} / \theta_{\mathrm{dm}, \mathrm{NC}}$.

For the DMvel class II model (case $b)$ ), there are only tiny changes to the velocity and lensing signals. While there is a clear divergence of the dark matter velocity and overdensity field in the DEvel class II model (case $c$ )), we must be careful in considering how to apply observational constraints to this model. This enhancement of the velocity (for $\xi$ negative) yields the fifth force effect which is only present in DEvel models (see also the $(1-b)$ term in Eq. (2.15)). This effect will be described in more detail in $§ 4.3 .1$.

In summary, in both the DMvel and the DEvel models the dark coupling can, in principle, be distinguished from a generic, uncoupled, dark energy described by $\tilde{w}(z)$ with perturbations growing as in standard GR. Note however that in the DMvel model GR is not modified. Although extremely small in this example, this class of models displays a mis-match between the reconstructed expansion history and the growth of structures, representing an exception to the commonly accepted interpretation that such a mis-match would be a tell-tale sign of modification of GR. In other words, if the measured expansion history and growth of structure are in agreement, this observation can be used to constrain deviations from GR. The conversely however is not necessarily true: a mis-match between expansion and growth could indicate deviations from GR or a dark coupling with GR unchanged.

\section{Low Redshift Observational Probes of Dark Coupling}

Coupled cosmologies, in order to fit the high-precision CMB data, predict values for the present-day cosmological parameters that differ substantially from the parameters values within non-interacting cosmologies. In other words, coupled cosmologies, in order to fit the high redshift (high $z$ ) observations, yield different expansion histories and different (shifted) values for present-day cosmological parameters. We show how to detect the induced shifts in the present-day value of some cosmological key parameters, as the Hubble constant $H_{0}$. Growth of structure probes are also explored and shown to be a powerful tool: while current errors on local bulk flows are still large, forecasted constraints on peculiar velocities offer a promising avenue. Let us mention that all the numerical results below were obtained choosing the cosmological parameters (as, for instance, $\Omega_{(0)} \mathrm{dm} h^{2}$ ) accordingly to WMAP 5 year angular diameter distance data [1] as explained in section 3 for the Class II models

studied here. For Class I DMvel models these numbers are provided in Ref. [26]. Also, changes in e.g. last scattering surface, matter-radiation equality, and growth history for 

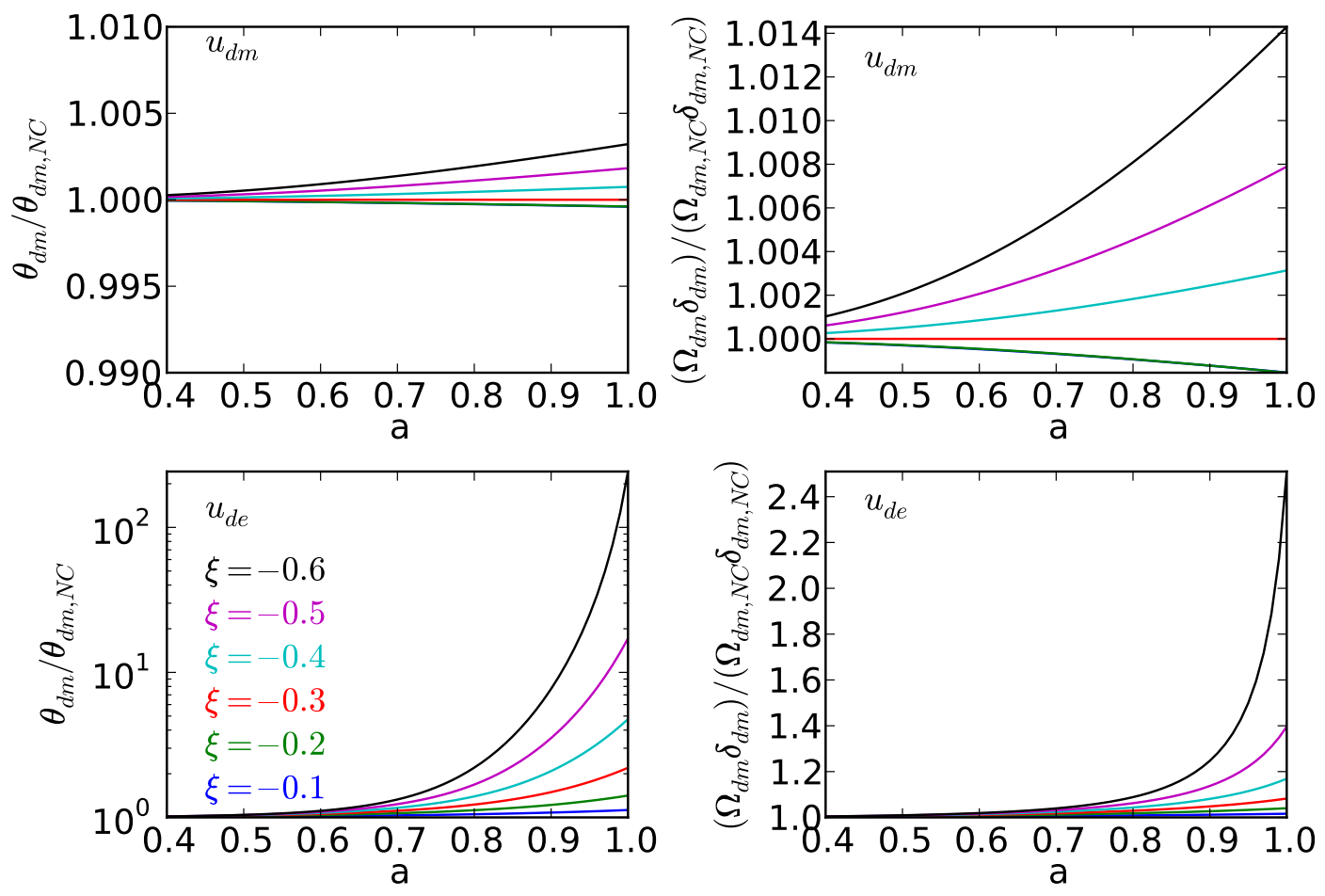

Figure II: The ratio of the amplitude of dark matter peculiar velocities, $\theta_{\mathrm{dm}}$, and the lensing signal, $\Omega_{\mathrm{dm}} \delta_{\mathrm{dm}}$ in coupled models compared to models with standard GR growth with identical expansion histories (labelled with $\mathrm{NC}$ for "Not Coupled"). The curves show $\xi=0,-0.1, \ldots-0.6$. The upper left panel shows the velocity ratio in the DMvel model and the upper right panel shows the lensing ratio. The two bottom panels show the same but for the DEvel model.

cosmological perturbations are fully taken into account by means of a modified version of CAMB [68] which includes coupling effect at the background and linear perturbation level.

\subsection{Background High-z vs Low-z quantities mis-match: a worked example with Hubble constant measurements}

We first consider future CMB data (Planck) and future constraints on $H_{0}$ (constraints from present cosmological data were already considered in Ref. [24] using the cosmomc package [69]). As it is often the case for forecasting the errors on parameters achievable with future data we make use of the Fisher matrix approach.

Let us focus on the DMvel class II dark coupled models given by $Q=\xi \mathcal{H} \rho_{\text {de }}$ (i.e. the first equation of Eqs. (2.22)) and calculate the shifts induced by the (neglected) coupling $\xi$ on the present-day value of the different cosmological parameters. We apply the technique of Ref. [70] to compute the expected shifts at zero redshift on the cosmological parameters due to the presence of non-zero coupling in the data and of setting coupling to zero in the analysis. The authors of [70] developed a method which exploits the Fisher information matrix formalism. We briefly summarize their method in the context of interacting 
models. Let us assume that we have two competing models: the $M^{\prime}$ model (a $\Lambda$ CDM universe) versus the $\mathrm{M}$ model (the DMvel coupled cosmology considered here). The method assumes that the $n^{\prime}$ parameters of model $M^{\prime}$ are common to $M$, which has $p=n-n^{\prime}$ extra parameters in it. These extra parameters are fixed to fiducial values in $M^{\prime}$. For the form of dark coupling we illustrate here (the application to other coupled models is straightforward), $p=1$, being the extra parameter the dimensionless coupling $\xi$ of the DMvel interacting model, which is set to $\xi=0$ in the $M^{\prime}$ model $(\Lambda \mathrm{CDM})$. If the true underlying model turns out to be the $M$ model (coupled cosmology), in the $M^{\prime}$ model, the maximum of the expected likelihood will not be at the correct values of the parameters. The $n^{\prime}$ parameters shift from their true values to compensate the fact that, effectively, the coupling $\xi$ additional parameter is being kept at an incorrect fiducial value (i.e. is set to zero). If this (incorrect) fiducial value differs by $\delta \phi_{\xi}$ from its true value, the others are shifted by an amount [70]

$$
\delta \theta_{\alpha}=-\left(F^{\prime-1}\right)_{\alpha \beta} G_{\beta \xi} \delta \phi_{\xi}
$$

where $\alpha, \beta=1 \ldots n^{\prime}, F^{\prime}$ is the Fisher matrix for the model $M^{\prime}(\Lambda \mathrm{CDM})$ and $G_{\beta \xi}$ is a submatrix of the $M$ model (interacting cosmology) Fisher matrix. The set of $n^{\prime}$ parameters we use here to describe the $M^{\prime}$ model are the current baryon and dark matter energy densities $\Omega_{\mathrm{b}} h^{2}$ and $\Omega_{\mathrm{dm}} h^{2}$, the current value of the Hubble parameter $h$, the amplitude $A_{s}$ of the primordial scalar spectrum, the scalar spectral index $n_{s}$ and the equation of state of the dark energy component $w$. We have computed the shift in the $n^{\prime}$ parameters for a CMB experiment with the specifications of Planck ${ }^{7}$. The CMB Fisher matrix has been computed following Ref. [71] using a modified version of CAMB which includes the effect of the coupling at the background and linear order perturbation level. The Fisher matrix in the $M^{\prime}$ cosmology contains six parameters: $\Omega_{\mathrm{dm}} h^{2}, \Omega_{b} h^{2}, H_{0}, n_{\mathrm{s}}, w$ and $A_{\mathrm{s}}$, being $A_{\mathrm{s}}$ is the amplitude of the primordial spectrum and $n_{\mathrm{s}}$ the spectral index. The Fisher matrix in the coupled cosmology will contain an extra parameter, the dimensionless coupling $\xi$.

If we were living in a coupled Universe and future CMB Planck data were to be (wrongly) fitted to a $\Lambda \mathrm{CDM}$ cosmology, the value of $H_{0}$ would shift by $d \ln H_{0} / d \xi=0.3$. This is the result of our Fisher analysis. However if the CMB data were to be fitted to a model where $w$ is also a free parameter, new degeneracies open up and $d \ln H_{0} / d \xi$ increases. Thus if the Hubble constant $H_{0}$ is determined independently with an uncertainty below $3 \%$, small values of coupling could be ruled out. A number of experiments (HST, Spitzer, GAIA and JWST [72]) are expected to measure $H_{0}$ with $2 \%$ uncertainty in the next decade. Consequently, coupled models could be highly disfavoured over the next decade by the combination of CMB Planck data with precise measurements of the Hubble constant $H_{0}$. The other cosmological parameters will also be shifted from their true values but by amounts expected to be smaller than their combined statistical and systematic errors.

The shifts for $H(z)$ (at $z>0$ ), as one may expect, are maximal at $z=0$, thus forthcoming $\mathrm{BAO}$ observations which constrain $H(z)$ with $\sim \%$ precision but only at a $z$ where the volume element per unit solid angle is near maximal, will not offer significant further improvement.

\footnotetext{
${ }^{7}$ www.rssd.esa.int/PLANCK
} 
To conclude, in this section we have provided quantitative forecasts of how future constraints on $H_{0}$ can help in constraining the coupling between the dark sectors.

\subsection{Effects on the skewness}

It is well known that the skewness of the density field is relatively insensitive to the expansion history (and thus the cosmological background) but is very sensitive to the growth of structure: mildly non-linear gravity leaves a distinct signature on the three-point correlation function (and therefore on the skewness) and galaxy bias also alters the skewness of the galaxy density field compared with the skewness predicted for the dark matter distribution. Here we consider the skewness as a test for the effects of dark coupling, following Refs. [20,73]. A large effect on the skewness is a clear indication that the growth of perturbations is modified with respect to the standard expectation. Skewness tests are also affected by the ambiguity of whether galaxies trace the baryon or the dark matter distribution.

Skewness is defined as the third order moment of the galaxy distribution

$$
S_{3}=\frac{<\delta(x)^{3}>}{\left(<\delta(x)^{2}>\right)^{2}}
$$

where $\delta(x)$ is the density contrast at the point $x$. In an Einstein de Sitter cosmology the skewness is predicted to be $34 / 7$. This value has been shown to be independent of the nature of the dark energy component [74] or the background cosmology $[75,76]$ and it is only very mildly dependent on the shape of the primordial power spectrum (given current constraints on the spectral index). Here, we study possible deviations of the skewness from its standard value in the context of coupled cosmologies. Following Ref. [74] we compute the perturbation evolution in second order perturbation theory in coupled cosmologies, and compute the skewness parameter as

$$
S_{3}=4+2 \epsilon=\frac{34}{7}+\frac{6}{7}\left(\frac{7}{3} \epsilon-1\right)
$$

where $\epsilon$ is a function of the first and second-order time-dependent components of density contrast $\delta_{(1)}(t)$ and $\delta_{(2)}(t)$, and reads

$$
\epsilon=\frac{\delta_{(2)}(t)}{\delta_{(1)}^{2}(t)} .
$$

$\delta_{(1)}(t)$ is the solution of the growth equation at first order in perturbation which corresponds to Eq. (2.19) for uncoupled or DMvel class I models and to Eq. (3.3) for DMvel \& DEvel class II considered here. Note, as we illustrate in the following, that the evolution equation

for the second order density perturbation $\delta_{(2)}(t)$ for the matter fluids $\alpha=\mathrm{dm}$, b depends as well on $\delta_{(1)}(t)$.

Let us first analyze the case of $Q \propto \rho_{\mathrm{dm}}$ (class I) models. Ref. [73] has shown that the skewness deviations in DEvel class I cosmologies are $\sim 1 \%$ for values of the coupling parameter satisfying current CMB constraints. For uncoupled and DMvel class I interacting 
models, the equation for the second order density perturbation evolution for the matter fluids $\alpha=\mathrm{dm}$, b reads

$$
\delta_{(2), \mathrm{dm}}^{\prime \prime}=-(2-q) \frac{\delta_{(2), \mathrm{dm}}^{\prime}}{a}+\frac{3}{2}\left[\Omega_{\mathrm{dm}}\left(\frac{\delta_{(2), \mathrm{dm}}}{a^{2}}+\frac{\delta_{(1), \mathrm{dm}}^{2}}{a^{2}}\right)+\Omega_{\mathrm{b}}\left(\frac{\delta_{(2), \mathrm{b}}}{a^{2}}+\frac{\delta_{(1), \mathrm{dm}} \delta_{(1), \mathrm{b}}}{a^{2}}\right)\right] .
$$

For the DMvel class I interacting model of Eq. (2.23), the deviations of the skewness for both the cold dark matter and baryon distributions from its standard value is smaller than $1 \%$ for the WMAP best fit of Ref. [26] and thus hard to be measured, even with upcoming future galaxy surveys. Recall that for DMvel class I models the continuity and Euler equations are exactly the same as for non interacting cases (after neglecting dark energy perturbations), and the only change in the growth is only introduced via the modified background evolution of $\mathcal{H}$ and $\rho_{\mathrm{dm}}[25,38]$.

For the DMvel and DEvel coupled class II models with $Q=\xi \mathcal{H} \rho_{\text {de }}$ of Eqs. $(2.22)$, the second order growth equation for the dark matter fluid in the Newtonian limit is given by:

$$
\delta_{(2), \mathrm{dm}}^{\prime \prime}=-B \frac{\delta_{(2), \mathrm{dm}}^{\prime}}{a}+\frac{3}{2}\left[\Omega_{\mathrm{dm}}\left(\frac{A \delta_{(2), \mathrm{dm}}}{a^{2}}+\frac{\delta_{(1), \mathrm{dm}}^{2}}{a^{2}}\right)+\Omega_{\mathrm{b}}\left(\frac{\delta_{(2), \mathrm{b}}}{a^{2}}+\frac{\delta_{(1), \mathrm{dm}} \delta_{(1), \mathrm{b}}}{a^{2}}\right)\right],
$$

where $A$ and $B$ are given by Eqs. (3.5) and (3.4) respectively. Let us recall that in order to obtain the growth equations for $Q=\xi \mathcal{H} \rho_{\text {de }}$ models we have neglected the contributions of $\delta_{Q}$. The additional terms proportional to $\xi \delta, \xi \delta^{\prime}$ in Eqs. (3.3) and (4.6) yield large effects on the cold dark matter distribution, and we observe $\sim 10 \%$ deviations in the dark matter skewness. For the baryon distribution, the effects are below $1 \%$.

As already pointed out in $\S 3$, for these two DMvel and DEvel class II models, the cold dark matter perturbation evolution can differ significantly from the baryon evolution, i.e. the changes in the growth of perturbations at linear and second order in these two models are not just due to a different background evolution. Even if the skewness effects in the cold dark matter distribution for these interacting models are large, it is not clear if they can be measured by galaxy surveys. Remember that in $\Lambda$ CDM baryons and dark matter interact gravitationally in the same way at large scales so that the problem does not appear.

If observations of the skewness of the galaxy distribution probe the baryon distribution, then skewness tests are not useful in constraining the dark coupling. Indeed, the presence of the coupling term only give rise to a $1 \%$ effect which is small compared with any foreseeable error-bar. If skewness observations (maybe via weak lensing, although recall that weak lensing probes the combination $\delta_{\mathrm{dm}} \Omega_{\mathrm{dm}}$ and not $\delta_{\mathrm{dm}}$ or $\Omega_{\mathrm{dm}}$ alone) can yield the dark matter skewness, then this approach may be useful given the expected $10 \%$ effect.

\subsection{Peculiar Velocities}

The velocity field offers a test of the growth of structure, which is complementary to galaxy clustering, as, for example, is less sensitive to non-linearities and bias. Below we consider constraints that can be obtained from present bulk flow data in the local universe 
and forecasted constraints achievable from future galaxy redshift surveys. These forecasts should be considered complementary to those presented in Refs. [24,26,28-35]. Throughout this section we will fix the CMB observables angular diameter distance to the last scattering surface and the physical dark matter and baryon density at decoupling.

\subsubsection{Constraints from peculiar velocities: Local bulk flows}

Recently Watkins et al. [77] have reported the observation of anomalously large bulk flows on $100 h^{-1}$ Mpc scales. In a Gaussian window of radius $50 h^{-1} \mathrm{Mpc}$, they find a coherent bulk motion of $407 \pm 81 \mathrm{~km} / \mathrm{s}$ in conflict with the $\Lambda$ CDM expectation of $\sim 200 \mathrm{~km} / \mathrm{s}$ at the $2 \sigma$ level. Reference [78] pointed out that these results, if confirmed, would favour models with a growth of perturbations larger than $\Lambda \mathrm{CDM}$ model predictions.

We first clarify the relation between the density and velocity fields in a general context where coupling is allowed. We again adopt a CMB-centric view: the amplitude of fluctuations at the epoch of recombination are well-measured, and so in the analysis that follows, we consider those to be fixed. We denote the amplitude of the density perturbation $A_{\mathbf{k}}$ for a particular $\mathbf{k}$-mode. The solution of the linear perturbations evolution (Eqs. (2.12) $(2.17))$, which we refer to by $\tilde{\theta}_{\mathrm{dm} / \mathrm{b}}(z)$ and $\tilde{\delta}_{\mathrm{dm} / \mathrm{b}}(z)$, describe the redshift evolution of the amplitude of each independent $\mathbf{k}$ mode:

$$
\begin{aligned}
& \delta_{\mathbf{k}, \mathrm{dm} / \mathrm{b}}(z)=A_{\mathbf{k}} \frac{\tilde{\delta}_{\mathrm{dm} / \mathrm{b}}(z)}{\tilde{\delta}_{\mathrm{dm} / \mathrm{b}}\left(z_{\mathrm{CMB}}\right)} \\
& \theta_{\mathbf{k}, \mathrm{dm} / \mathrm{b}}(z)=A_{\mathbf{k}} \frac{\tilde{\theta}_{\mathrm{dm} / \mathrm{b}}(z)}{\tilde{\theta}_{\mathrm{dm} / \mathrm{b}}\left(z_{\mathrm{CMB}}\right)},
\end{aligned}
$$

where we have emphasized that when coupling is present, the growth of baryon and matter density and velocity perturbations are not necessarily the same. In the uncoupled case in the Newtonian limit $(k \gg \mathcal{H})$ and assuming that $\hat{c}_{s \text { de }}^{2}=1$, baryon and matter perturbations trace each other, and the velocity and density perturbations are related by:

$$
\theta_{\mathbf{k}, \mathrm{dm} / \mathrm{b}}=-a H \frac{A_{\mathbf{k}}}{\tilde{\delta}_{\mathrm{dm} / \mathrm{b}}\left(z_{\mathrm{CMB}}\right)} \frac{\partial \tilde{\delta}_{\mathrm{dm} / \mathrm{b}}}{\partial \ln a} \text { (uncoupled) } .
$$

In general, the relation between the growth equation solutions $\tilde{\delta}_{d m, b}$ and $\tilde{\theta}_{d m, b}$ is not so simple. Nevertheless, one can eliminate the original mode amplitude $A_{\mathbf{k}}$ by defining

$$
\tilde{f}_{\mathrm{dm}, \mathrm{b}}(z) \equiv-\frac{\tilde{\theta}_{\mathrm{dm}, \mathrm{b}}(z) / \tilde{\theta}_{\mathrm{dm}, \mathrm{b}}\left(z_{\mathrm{CMB}}\right)}{a H \tilde{\delta}_{\mathrm{dm}, \mathrm{b}}(z) / \tilde{\delta}_{\mathrm{dm}, \mathrm{b}}\left(z_{\mathrm{CMB}}\right)} .
$$

In the non-coupled case, $\tilde{f}=\partial \ln \tilde{\delta} / \partial \ln a$ (the same for baryons and dark matter), while in class II models $\left(Q \propto \rho_{\mathrm{de}}\right)$ for both DEvel and DMvel, $\tilde{f}_{\mathrm{dm}}=\partial \ln \tilde{\delta}_{\mathrm{dm}} / \partial \ln a+\xi \rho_{\mathrm{de}} / \rho_{\mathrm{dm}}$. Finally, we can write the linear velocity field in terms of the density field as

$$
\vec{v}_{\mathrm{dm}, \mathrm{b}}(k, a)=\frac{i \tilde{f}_{\mathrm{dm}, \mathrm{b}} a H \delta_{\mathrm{dm}, \mathrm{b}}(k, a) \vec{k}}{k^{2}} .
$$


Following Ref. [78], the expected mean square velocity is

$$
\begin{aligned}
\left\langle u^{2}\right\rangle & =\frac{1}{2 \pi^{2}} \int_{0}^{\infty} \mathrm{d} k k^{2} P_{v}(k)|\tilde{W}(k)|^{2} \\
& =\frac{\tilde{\theta}(z)^{2}}{2 \pi^{2} \tilde{\theta}^{2}\left(z_{\mathrm{CMB}}\right)} \int_{0}^{\infty} \mathrm{d} k\left\langle A_{\mathbf{k}} A_{\mathbf{k}}^{\star}\right\rangle|\tilde{W}(k)|^{2},
\end{aligned}
$$

where $P_{v}(k)$ is the peculiar velocity power spectrum and $\tilde{W}(k)$ is the Fourier transformed spherically symmetric window function ${ }^{8}$. In the second equality, the term outside the integral depends only on the normalized growth of velocity perturbations, $\tilde{\theta}(z) / \tilde{\theta}\left(z_{\mathrm{CMB}}\right)$, while the value of the integral depends on the power spectrum of initial fluctuations. ${ }^{9}$ This formula shows that bulk flows would be larger if the growth of perturbations is enhanced by some mechanism. In coupled dark matter-dark energy cosmologies, the growth factor can be modified in several ways [38] and therefore the predictions for the bulk flow may differ from those obtained in the context of a $\Lambda \mathrm{CDM}$ universe. Here we explore if coupled models could be favoured by bulk flow measurements.

For the DMvel $Q \propto \rho_{\mathrm{dm}}$ interacting model of Eq. (2.23) both the continuity and Euler equations are unchanged and therefore, the expression for the uncoupled velocity amplitude $f=\partial \ln \tilde{\delta} / \partial \ln a$ will still hold.

Ref. [26] showed that in this model, CMB data alone disfavor positive values of $\Gamma$ but still allow large negative values. For their $\mathrm{CMB}$ best fit model with $\Gamma / H_{0}=-0.3$, we obtain a bulk flow of $171 \mathrm{~km} / \mathrm{s}$. In principle, positive, higher values of $\Gamma$ will predict larger values for the bulk flows. However, these values of $\Gamma$ would not provide a good fit to $\mathrm{CMB}$ and/or BAO and SN data, see the analysis presented in Ref. [26]. Therefore, bulk flow observations do not indicate a preference for this particular coupled model.

Figure III shows the bulk flow results for the DMvel and DEvel class II models $(Q \propto$ $\left.\rho_{\text {de }}\right)$ as a function of the coupling $\xi$. The concordance $\Lambda$ CDM cosmology predicts a bulk flow of $\left\langle u^{2}\right\rangle^{1 / 2}=203 \mathrm{~km} / \mathrm{s}$. As we have seen in $\S 3$, the DMvel model has dark matter peculiar velocities which are similar to those of non interacting models, since the Euler equation is unmodified. This is not the case for the DEvel model, in which the Euler equation changes, as can be seen from Fig. III: values of the coupling $\xi<-0.35$ lead to an effective $3 \sigma$ level deviation from observations.

However, this model must be handled with care since the dark matter and baryon perturbations are not equal. This large bias between the dark matter, baryon peculiar velocities and overdensities was shown in $\S 3$. Though smaller in amplitude, this feature is also seen in the coupled quintessence model explored with $N$-body simulations in Ref. $[79,80]$. While it is at first unclear which field the galaxies trace, we argue here that for $\xi>-1$ the velocities probed by, e.g., luminous red galaxies should be those of the dark matter, at least in the class II model considered in $\S 3$. It is generally accepted that the

\footnotetext{
${ }^{8}$ For our numerical calculations, we use a Gaussian window function $\tilde{W}(k)=e^{-k^{2} R^{2} / 2}$ of radius $R=$ $50 h^{-1} \mathrm{Mpc}$.

${ }^{9}$ This is not precisely true since $\left\langle A_{\mathbf{k}} A_{\mathbf{k}}^{\star}\right\rangle$ is constrained tightly by the CMB in physical units $(k$ in $\mathrm{Mpc}^{-1}$ ), while the radius of the window function is fixed at $50 h^{-1} \mathrm{Mpc}$. We account for this dependence on $h$ in our calculations.
} 


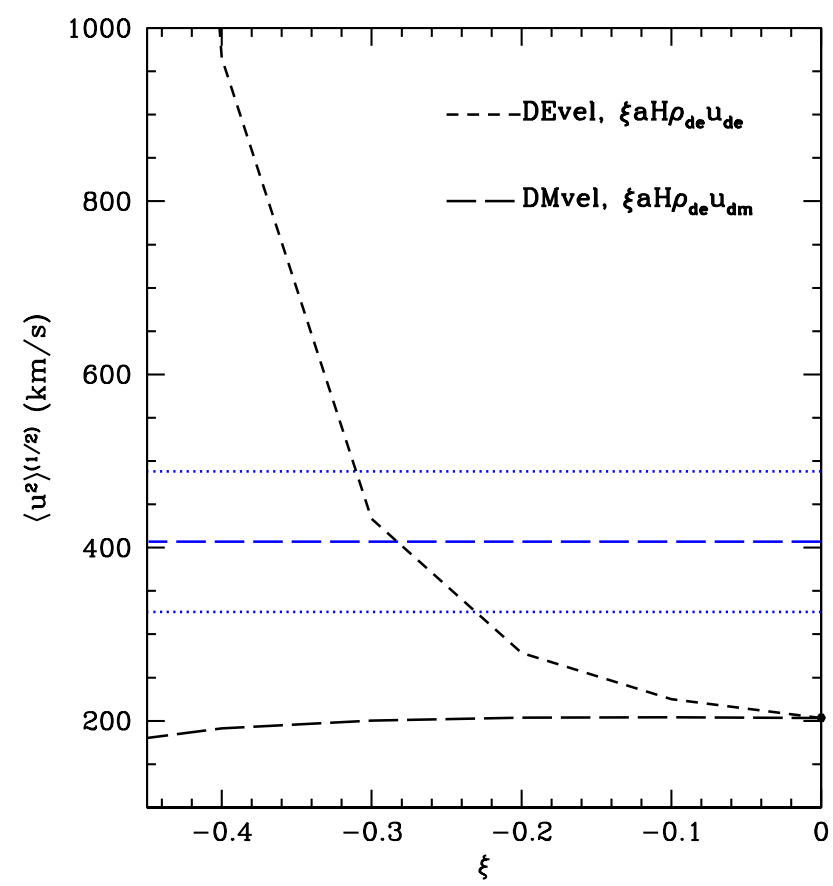

Figure III: The short (long) dashed curve shows the bulk flow predictions for the DEvel (DMvel) class II interacting model with $Q=\xi \mathcal{H} \rho_{\mathrm{de}}$. The cosmological parameters have been chosen to fit WMAP 5 year angular diameter distance data [1]. The dashed (dotted) blue lines represent the measured value of $407 \pm 81 \mathrm{~km} / \mathrm{s}$, and the circle depicts the $\Lambda \mathrm{CDM}$ model prediction for the bulk flows $\left\langle u^{2}\right\rangle^{1 / 2}=203 \mathrm{~km} / \mathrm{s}$.

massive luminous red galaxies (LRGs) were in place by redshift $z>1$ (see Refs. [81-88] and references therein). At this redshift, the energy-momentum exchange is negligible. Therefore, galaxy formation should proceed as expected in $\Lambda \mathrm{CDM}$ - in the potential wells of dark matter halos. Moreover, we know that luminous red galaxies occupy massive dark matter halos today from weak lensing measurements [89].

To demonstrate that these galaxies remain bound in the potential wells of their dark matter halos, we need to demonstrate that the differential acceleration of the dark matter and baryons due to the WEPV "fifth force" in this model is much smaller than the force binding the galaxy to the dark matter halo. As discussed in $\S 2.1$, the choice of DEvel and $Q \propto \rho_{d e}$ implies a fractional increase in the dark matter peculiar velocity equal and opposite to the fractional change in energy density as energy is transferred from dark matter to dark energy. This corresponds to an acceleration on a dark matter halo with peculiar velocity $v$ as

$$
\left(\frac{d v}{d t}\right)_{\mathrm{WEPV}}=v \times \frac{1}{m} \frac{d m}{d t}
$$

A dark matter halo has $\rho_{\text {halo }} \sim 200 \rho_{\text {crit }}$, so even for large couplings that correspond to depleting all of the mean dark matter density $\bar{\rho}_{\mathrm{dm}}$ in the universe in the past $1 \mathrm{Gyr}$, $1 / m d m / d t<1 / 200 \mathrm{Gyr}^{-1}$. We take $v$ to be the expected $\mathrm{rms}$ linear theory bulk flow, 
$\sim 500 \mathrm{~km} / \mathrm{s}$, and find $\left(\frac{d v}{d t}\right)_{\mathrm{WEPV}}<8 \times 10^{-14} \mathrm{~m} \mathrm{~s}^{-2}$. For a normal Newtonian orbit, the gravitational acceleration is

$$
\left(\frac{d v}{d t}\right)_{\text {gravity }}=\frac{G M}{r_{\mathrm{eff}}^{2}}=\frac{G M}{r_{\mathrm{vir}}^{3}} \frac{r_{\mathrm{vir}}}{\kappa^{2}} \sim \frac{100 H_{0}^{2} r_{\mathrm{vir}}}{\kappa^{2}},
$$

where $M$ is the mass of the halo and $r_{\text {eff }}$ is some fraction $\kappa<1$ of the virial radius $r_{\text {vir }}$ of the halo. For $r_{\text {vir }} \sim 0.5 \mathrm{Mpc}$ appropriate for these galaxies, we get $\left(\frac{d v}{d t}\right)_{\text {gravity }}>100\left(\frac{d v}{d t}\right)_{\mathrm{WEPV}}$. We conclude that under these assumptions, the galaxy peculiar velocities will trace the dark matter peculiar velocity field, and galaxy peculiar velocity observational results can be generally applied to interacting (DMvel or DEvel) class I and class II models.

\subsubsection{Constraints from peculiar velocities: redshift space distortions}

Even in the linear regime, peculiar velocities make the linear galaxy redshift-space power spectrum $P_{\mathrm{S}}(\mathbf{k})$ anisotropic when the underlying real space linear power spectrum $P_{\mathrm{dm}}(k)$ is isotropic. These anisotropies go under the name of redshift-space distortions. In linear theory and under the flat-sky, distant observer approximation, we can generalize the results of $[59,90]$ to coupled models by simply replacing $f \equiv \partial \ln \tilde{\delta} / \partial \ln a$ with $\tilde{f}$ defined in Eq. (4.10):

$$
\delta_{\mathrm{s}}^{\mathrm{gal}}(\mathbf{k}, z)=\left(b_{\mathrm{gal}}+\tilde{f} \mu^{2}\right) \delta_{\mathrm{dm}}(k, z),
$$

where $\mu$ is the cosine of the angle between the wave vector $\mathbf{k}$ and the line of sight of a distant observer, $b_{\text {gal }}$ the bias relating galaxy with dark matter overdensities in real space, i.e. $b_{\text {gal }} \equiv \delta_{\mathrm{g}} / \delta_{\mathrm{dm}}$, and $\delta_{\mathrm{dm}}(k, z)$ is the real space (isotropic) linear dark matter overdensity. Note that we assume in this section as well as in the previous one that galaxies trace the dark matter velocity fields rather than that of the baryons, since galaxies reside in dark matter halos. Therefore, in the notation of the previous section, galaxy redshift surveys constrain $\tilde{f} \delta_{\mathrm{dm}}=\left(\tilde{\theta}_{\mathrm{dm}}(z) / \tilde{\theta}_{\mathrm{dm}}\left(z_{C M B}\right) a H\right)\left\langle A_{\mathbf{k}} A_{\mathbf{k}}^{\star}\right\rangle^{1 / 2}$ using the $\mu$ dependence in Eq. (4.16). Note that in the literature (e.g., [59]) the redshift space distortions constraint is often written instead as $f \sigma_{8}$, even though this probe is only sensitive to the amplitude of the velocity field, and not the amplitude of density perturbations directly. We follow this labelling convention, but replacing $f \rightarrow \tilde{f}$ to account for the effects of dark coupling. Since coupled cosmologies can modify the growth of density and velocity perturbations in a significant way, one can use the measurements of redshift space distortions to constrain the strength of the dark sector interaction.

We first consider the DMvel class I coupled model of Eq. (2.23). For this form of coupling, as previously stated, both the continuity and Euler equations remain unchanged and in this case $\tilde{f}=\partial \ln \delta / \partial \ln a$. The only imprint of the dark sector interaction in the linear growth of perturbations for this first interacting model will therefore arise from the background quantities $\mathcal{H}$ and $\rho_{\mathrm{dm}}[25,38]$.

We also consider here the DMvel and DEvel class II interacting cosmologies of Eqs. (2.22). For these two cosmologies, the linear growth of the matter and velocity overdensities have extra contributions and therefore, the changes in the redshift space distortions observable will not arise exclusively from background quantities. 
The results of $\S 3$ indicate that the impact of coupling on velocity perturbations will predominantly be through the change in background in DMvel models, while sufficiently large couplings cause exponential growth of velocity perturbations in the DEvel model.
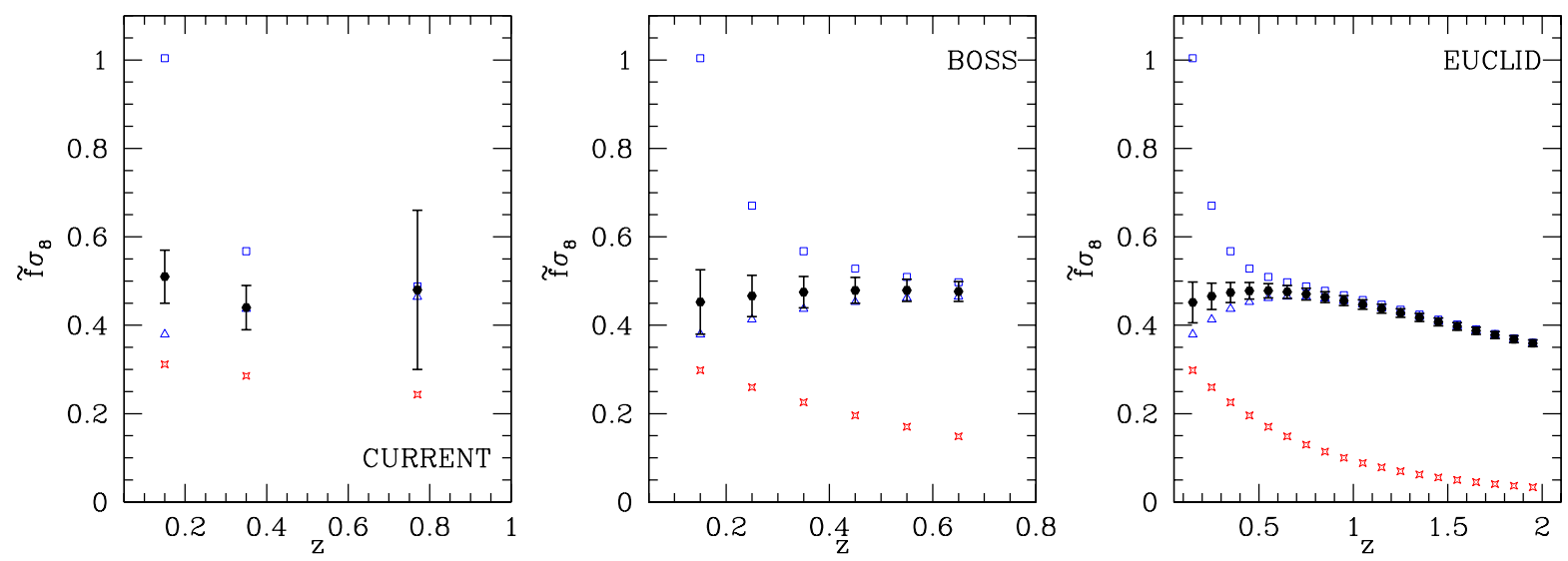

Figure IV: The red crosses denote the $\tilde{f} \sigma_{8}$ values for the model of Eq. (2.23), for $\Gamma / H_{0}=-0.3$ and cosmological parameters fixed to model 2 of Ref. [26]. The blue triangles (squares) depict the $\tilde{f} \sigma_{8}$ expectations for a DMvel (DEvel) class II model with $Q=\xi \mathcal{H} \rho_{\mathrm{de}}$ for $\xi=-0.5$. The points in the left panel show current measurements of $\tilde{f} \sigma_{8}$. The middle and right panels show the expected $\tilde{f} \sigma_{8}$ errors for BOSS and EUCLID-like surveys, respectively, assuming a fiducial $\Lambda$ CDM cosmology.

Figure IV illustrates the current and future constraining power of this method, where the red crosses show the $\tilde{f} \sigma_{8}$ predictions for the DMvel class I coupled model of Eq. (2.23), for $\Gamma / H_{0}=-0.3$, where the cosmological parameters have been fixed to their WMAP 5 year data best fit values (see model 2 of Ref. [26]). The blue triangles (squares) refer to the predictions of the DMvel (DEvel) class II model explored here, with $\xi=-0.5$ and $w=-0.9$ (the remaining cosmological parameters are chosen accordingly to WMAP 5 year angular diameter distance data [1]). The black circles in the first panel of Fig. IV represent current $\tilde{f} \sigma_{8}$ measurements [91], in the other two panels they show the $\tilde{f} \sigma_{8}$ forecasts for BOSS and EUCLID-like galaxy surveys, assuming a $\Lambda$ CDM fiducial cosmology. In order to forecast the errors from these two BAO galaxy surveys, we have used the Fisher information matrix formalism, combining the results from these two experiments with those expected from the on-going CMB Planck experiment. The Planck CMB Fisher matrix contains seven parameters

$$
\Omega_{\mathrm{dm}} h^{2}, \Omega_{\mathrm{b}} h^{2}, H_{0}, n_{\mathrm{s}}, w, A_{\mathrm{s}}, n_{\mathrm{run}},
$$

where $w$ is the dark energy equation of state, $A_{\mathrm{S}}$ is the amplitude of the primordial spectrum, $n_{\mathrm{s}}$ is the spectral index and $n_{\text {run }}$ is the spectral index running. The galaxy survey Fisher matrix contains one additional parameter, the growth factor $\tilde{f}$, plus a galaxy bias which is redshift dependent. We have marginalized over the galaxy bias in each redshift bin.

BOSS-like survey parameters are the following: redshift coverage $0<z<0.7, A_{\text {sky }}=$ $10000 \mathrm{deg}^{2}$ and a mean galaxy density of $2.66 \times 10^{-4}$. For the EUCLID-like survey we 
assume a redshift coverage of $0.15<z<2$, a sky area of $f_{\text {sky }}=20000 \mathrm{deg}^{2}$ and a mean galaxy density of $1.56 \times 10^{-3}$. We have verified that our errors are fully consistent with those obtained by means of the Fisher routine provided by Ref. [92].

Firstly, note from Fig. IV that, for the values of the parameters chosen in this figure, the $\tilde{f} \sigma_{8}$ values for the DMvel class I model of Eq. (2.23) are much lower than current estimations. As carefully explored in Ref. [25], negative values of $\Gamma$ (corresponding to positive $Q$ ) show a suppression of structure growth due to a smaller amount of dark matter in the past. The quantity $\tilde{f} \sigma_{8}$ will get depleted then, not only due to a lower growth factor $\tilde{f}$, but also due to a lower $\sigma_{8}$. However, current errors on $f \sigma_{8}$ measurements are large and do not rule out these models at a high significance level, and other cosmological parameter choices could differ in the $\tilde{f} \sigma_{8}$ predictions. Secondly, note that the predictions for the DMvel class II model are very close to those of a non interacting model, as expected from the results presented on dark matter velocities for this model in $\S 3$ and in $\S 4.3 .1$. Finally, we can observe from Fig. IV that for the DEvel class II model, huge $\tilde{f} \sigma_{8}$ values are predicted, especially at low redshifts, when dark energy starts to be dominant and the effect of the energy-momentum transfer starts to be important in the dark matter differential velocity equation. Such an effect can not be obtained in the two DMvel class I and class II models explored here because the Euler equation for the dark matter velocity is not modified in these interacting cosmologies. In the DEvel class II model, couplings $\xi<-0.4$ will be ruled out at more than $3 \sigma$ with existing $\tilde{f} \sigma_{8}$ data.

Accurate measurements of $\tilde{f} \sigma_{8}$ from future BAO surveys as BOSS or EUCLID may rule out significantly the form of couplings explored above, see the central and right panels of Fig. IV. For future surveys such as BOSS and EUCLID, the forecasted errors on the coupling $\xi$ for both the DMvel and DEvel class II models with $Q=\xi \mathcal{H} \rho_{\text {de }}$ can be as small as $\Delta \xi=0.02$ and $\Delta \xi<0.01$ respectively. These forecasted errors should be considered as optimistic, since in their derivation, we have assumed a perfect knowledge of the remaining cosmological parameters.

\subsection{Weak equivalence principle violation (WEPV) constraints}

Violation of the equivalence principle in the dark sector is a general feature of DEvel models. Regardless of the underlying physics responsible for this effect, it can modeled phenomenologically by attributing to dark matter particles a "fifth force" specified by its potential.

\subsubsection{Coupled scalar field as an example of DEvel $Q \propto \rho_{\mathrm{dm}}$ models}

Kesden and Kamionkowski (K\&K in the following) [93,94], analyzed the consequences of WEP violation for dark-matter on galactic scales, focusing on dark-matter dominated satellite galaxies orbiting much larger host galaxies. It was shown by running several Nbody numerical simulations that in the presence of a dark matter fifth force a pronounced asymmetry appears in the leading compared to the trailing tidal stream of the satellite galaxy. In summary, they inferred that a difference among dark matter and baryonic accelerations larger than $10 \%$ is severely disfavoured. They found that the leading-to-trailing ratio exceeds 0.5 for all simulations without a dark-matter force and never exceeds 0.2 for 
a dark-matter force with $4 \%$ the strength of gravity. From the number of simulations performed, one can conclude that a difference between dark matter and baryon accelerations larger than $4 \%$ corresponds to at least a $2 \sigma$ deviation in leading-to-trailing ratio. However a more quantitative statement cannot be made as the way their simulations span the parameter space for satellite interactions may not reproduce the same probability distribution as real interactions.

To make this statement more quantitative we review the case DEvel class I models of the form of Eq. (2.24) considered in e.g., [79, 80,95-99]. In these models the effective gravitational potential between cold dark matter particles is of the Yukawa-type. In the limit of massless dark energy interacting scalar field $\phi$, the strength of the gravitational interaction is corrected by a $\beta^{2}$ contribution and reads:

$$
G_{c c}=G_{N}\left(1+2 \beta^{2}\right)
$$

where $G_{N}$ denotes the Newton's constant and $\beta$ parameterizes the dimensionless dark coupling of Eq. (2.24) as well as the strength of WEPV. It should be stressed that in these coupled quintessence models, the correction to the standard gravitational potential term $k^{2} \Psi$ in the Euler equation for dark matter velocity perturbations is due to a contribution $\propto-\beta k^{2} \delta \phi$ where $\delta \phi$ is the scalar field perturbation. Indeed, it can be shown [20], that in these models $\beta \delta \phi \sim-\beta^{2} \Psi$, giving rise to a non negligible modification of gravity. Using Eq. (4.18), we see that the $10 \%$ limit from K\&K reduce to $|\beta|<0.22$ in our notation.

The authors of Ref. [100] obtained, in the notation used here, a $95 \%$ bound of $|\beta| \lesssim 0.11$ using CMB observations. More recently, it has been argued [101,102] that larger differences among dark matter and baryon accelerations, with an extra force as large as gravity, could be allowed if a screening length of $r_{s}=1 \mathrm{Mpc} / h$ is assumed. This screening allow the authors of Ref. [101,102] to evade cosmological-scales constraints (CMB). The caveat to this argument is that $\mathrm{CMB}$ constraints apply to redshift $z \simeq 1100$ and the parameter $\beta$ may vary with redshift. Ref. [103] find that large-scale structure observations at $z<0.2$ do not show any indication for a typical scale where this Yukawa-type modification switches on, but constrain the strength of the modification only weakly; [104] improve these bounds.

A possibly promising avenue to be explored from forthcoming large, SZ-selected galaxy clusters surveys, is that of the baryon mass fraction in galaxy clusters. In fact, as shown in [80], a WEPV, with increasing coupling reduces the clusters baryon fraction: a $\beta=0.2$ reduces $f_{\mathrm{b}}$ in clusters by $10 \%$ with respect of a $\Lambda \mathrm{CDM}$ model. This effect is mostly due to a mis-match of velocities on large-scales not driven by Eq. (4.18). Tantalizingly, this could alleviate the tension between cosmological and clusters determinations of $f_{\mathrm{b}}$ from current data, however to-date the error-bars are still large. The results of [80] derive from N-Body simulation for coupled models. This type of analysis had already been first performed in [79] and [80] claims to obtain similar results on the enhancement of the bias in the nonlinear region within and around massive halos. On a another hand, let us mention that the results of [80] completely disagree with those of [79] on the increase or decrease of the inner halo overdensity with respect to CDM assuming the existence of a coupling between the scalar field and dark matter. More constraints have been derived on these type of coupled models using background up to cosmological perturbations evolution (see 
e.g. $[98,99]$ ), and can affect the late-time Integrated Sachs-Wolfe (ISW) effect at large scales [105]. See also [106] for constraints on time dependent coupling.

Let us repeat that on cosmological scales, a WEPV only in the dark sector, would yield a mis-match between the baryon (and thus galaxy) distribution and the dark matter one [102] which is scale-dependent (see also [80]). By comparing left (weak lensing) and right (galaxy clustering) panel for fig. 2 of [104] we conclude that there is no evidence for a large mis-match from present data, however the error-bars are still large. Forthcoming gravitational lensing surveys, combined with galaxy and Lyman alpha surveys should therefore constrain these models further.

So far we have considered very specific models of the DEvel family for which a potential can be specified. The above consideration hold qualitatively also for the more phenomenologically-defined models as we show next.

\subsubsection{DEvel $Q \propto \rho_{\text {de }}$ model}

As an example, we illustrate also the effect of from WEPV for the DEvel class II toy-model considered along this paper and characterized by $Q_{\nu}=\xi H \rho_{\mathrm{de}} u_{\nu}^{\mathrm{de}}$. In this DEvel class II model still the dark matter and baryon accelerations are clearly different, i.e. compare Eq. (2.15) with $b=0$ to Eq. (2.13). The results obtained in the following for this particular choice of coupling can be easily generalized to other choices of interaction. At sub-horizon scales, in the Newtonian regime $(k \gg \mathcal{H})$, the baryon and dark matter accelerations are given by:

$$
\begin{aligned}
\dot{\theta}_{\mathrm{b}} & =\mathcal{H} \dot{\delta}_{\mathrm{b}}+k^{2} \Psi . \\
\dot{\theta}_{\mathrm{dm}} & =\mathcal{H}\left(1+\xi \frac{\rho_{\mathrm{de}}}{\rho_{\mathrm{dm}}}\right)\left(\dot{\delta}_{\mathrm{dm}}+\xi \mathcal{H} \delta_{\mathrm{dm}} \frac{\rho_{\mathrm{de}}}{\rho_{\mathrm{dm}}}\right)+k^{2} \Psi
\end{aligned}
$$

where we have neglected $\delta_{\mathrm{de}}$ and $\delta H$ contributions and $k^{2} \Psi$ reads

$$
k^{2} \Psi=-\frac{3}{2} \mathcal{H}^{2}\left(\Omega_{\mathrm{b}} \delta_{\mathrm{b}}+\Omega_{\mathrm{dm}} \delta_{\mathrm{dm}}\right) .
$$

Figure V shows the effect of the dark coupling on the relative dark matter-baryon acceleration for the model illustrated here, for two different assumptions about the dark energy equation of state $w$. The cosmological parameters (as, for instance, $\Omega_{\mathrm{dm}} h^{2}$ ) have been chosen accordingly to WMAP 5 year angular diameter distance data [1] as explained in section 3 .

There is clearly a well defined relation between the strength of coupling and the mismatch between the dark matter and baryon acceleration that is robust to changes in $w$ and is well approximated by a power law:

$$
\ln \left(\frac{\dot{\theta}_{\mathrm{b}}-\dot{\theta}_{\mathrm{dm}}}{\dot{\theta}_{\mathrm{b}}}\right)=2.69+0.977 \ln (-\xi)
$$

up to mis-matches of order of few $\times 100 \%$. This has been derived assuming linear theory and therefore quantitatively strictly applies only to cosmological linear scales. 


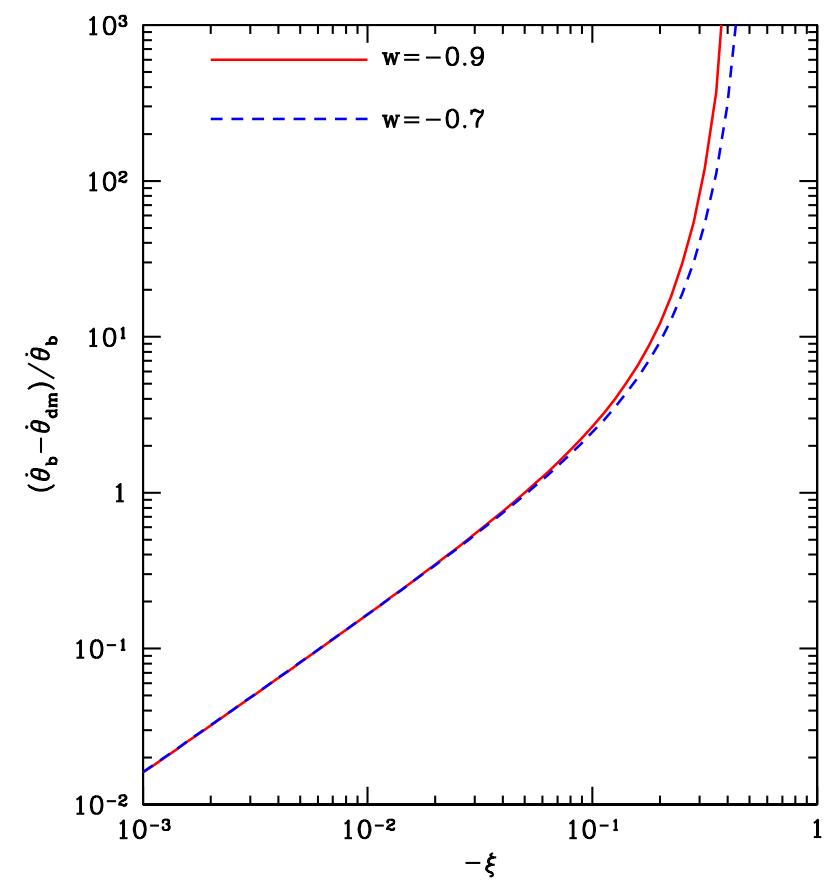

Figure V: Relative dark matter-baryon acceleration $\frac{\dot{\theta}_{\mathrm{b}}-\dot{\theta}_{\mathrm{dm}}}{\dot{\theta}_{\mathrm{b}}}$ for $Q_{\nu}=\xi \mathcal{H} \rho_{\mathrm{de}} u_{\nu}^{(\mathrm{de})} / a$ using Eq. (4.19) and (4.20). The cosmological parameters have been chosen to fit WMAP 5 year data, see text for more details. The red solid (blue short dashed) curve assumes an equation of state of the dark energy component $w=-0.9(w=-0.7)$.

From the discussion above, our numerical results seems to indicate that for DEvel class II models with $Q_{\nu}=\xi \mathcal{H} \rho_{\mathrm{de}} u_{\nu}^{\text {de }} / a$, couplings $\xi$ smaller than $\sim-0.002$ would lead to a $2 \sigma$ sigma deviation in the leading-to-trailing ratio and couplings smaller than $\sim-0.01$ are strongly disfavoured by K\& K numerical analysis. Let us mention that those results are to be taken with care given that no asymmetry in leading compare to the trailing of e.g. Sagitarius dwarf galaxy is observed while K\& K numerical best fit model tends to a leading-to-trailing ratio of 0.66 in the absence of any dark matter fifth force

\subsection{Constraints from matter abundance in galaxy voids}

An uncontroversial (indisputable) feature of these models arises from the fact dark energy is smoothly, uniformly distributed but dark matter is not, and most of the volume of the Universe is occupied by regions under-dense of dark matter (voids). The coupling $Q \propto \rho_{\text {de }}$ characteristic of class II models requires that, independently on the local dark matter density, a given amount of dark matter per unit volume must turn into dark energy. Thus in the underdense regions the coupling $Q \propto \rho_{\text {de }}$ must be just an effective, coarsegrained description: in fact, once locally in an underdense region all dark matter has been transformed into dark energy, the description adopted must break down. In addition, the uniform depletion of dark matter makes the overdensity in voids approach $\delta \sim-1$ very rapidly, implying a prompt breakdown of linear perturbation theory. While a detailed 
modeling of this behavior may require numerical simulations, it is clear that observations of underdense regions offer a promising avenue to constrain such models. We consider now observations of voids properties to constrain these models.

Coupled dark energy-dark matter models lead to a dark matter depletion (or enhancement, depending on the sign and form of the interacting term). Most of this depletion/enhancement will take place from the background, which in turns translates in depleting/enhancing the voids in the large scale structure, which themselves occupy the most volume. Therefore, any measurement of the amount of matter in the voids with respect to the standard non-coupled model can help to constrain the value of the coupling.

The matter content in voids has received recent attention (see e.g. Refs. [107,108] and references therein) because it seems that the voids are more empty than expected from $\Lambda$ CDM model predictions. The work developed in Ref. [108] summarizes very well our current knowledge on the occupancy of voids. The local group, mostly our galaxy and Andromeda in mass content, is about $20 \mathrm{Mpc}$ away from the nearest cluster of galaxies, Virgo, and sits in a relatively low-density region e.g., [109]. Ref. [108] argue that in the local volume, at a distance from us of about $10 \mathrm{Mpc}$, there are too few small galaxies with circular velocities $V_{c}$ below $\sim 35 \mathrm{~km} / \mathrm{s}$. They estimate a factor 10 discrepancy with the $\Lambda \mathrm{CDM}$ model predictions. Other authors [107] have previously developed models to clear the voids of dark matter and thus quench the number of dark halos that could host these small galaxies. Of course, another explanation is that the $\Lambda$ CDM model is correct and some galaxies are simply not visible [110,111]. Coupled models in which a given amount of dark matter per unit volume turns into dark energy, regardless of the local density would provide, therefore, a mechanism to clear dark matter (and galaxies) from the voids. On the other hand, the void phenomenon disfavors coupled models in which the dark matter content per unit volume is enhanced. As an illustration, we use recent void results [108] to derive constraints on DMvel and DEvel class II models which will lead to a depletion of the dark matter energy density of the universe, in particular, the dark coupled model of Eqs. (2.22) characterized by the dimensionless coupling $\xi$. The latter cannot be too large and negative to empty the voids completely because, some galaxies with circular velocities $V_{c}$ below $35 \mathrm{~km} / \mathrm{s}$ are still observed in voids.

Before getting a precise estimate on the value of $\xi$, one can already impose a preliminary lower bound on the coupling parameter by requiring the universe as a whole not to be empty of matter in the voids today. Figure I shows the current cold dark matter energy density in the universe as a function of the coupling for the dark sector interaction given by Eq. (2.22) necessary to fit WMAP 5 year data [1]. The effect of the WMAP parameters uncertainties is illustrated by the two lines, chosen to vary along the parameter-degeneracy that most affect this figure. This rather simple argument is indicating us that $\xi>-0.6$, see $\S 3$. In what follows we will improve this lower limit on $\xi$. To proceed, we will assume that dark matter halos associated to galaxies with circular velocities $\lesssim 35 \mathrm{~km} / \mathrm{s}$ are depleted by a factor of 10 as argued in [108]. Note that at circular velocities of $\sim 50 \mathrm{~km} / \mathrm{s}$ there is no discrepancy with a $\Lambda$ CDM model [112]. We further argue that such a small galaxies and their associated halos in low density regions form recently, when dark energy is important. In fact photo-ionization before recombination blows gas away from the halos of small mass. 
Only those small mass halos that collapse at $z<1$ are able to retain their gas and thus form stars (e.g. [113] and references therein). With this information we can compute the maximum allowed dark matter depletion from the local void and translate it into a bound on $\xi$. In fact the velocity function can be transformed into a mass function using the spherical collapse (i.e., $35 \mathrm{~km} / \mathrm{s}$ corresponds to $\sim 8 \times 10^{9} M_{\odot}$ ). At these small masses the velocity function and mass functions (both baryonic Ref. [86] and dark matter) are well approximated by power laws. We thus modify the $\Lambda$ CDM-predicted power law for the local volume mass function to match the factor of 10 depletion below $35 \mathrm{~km} / \mathrm{s}$, and still matching the $\Lambda \mathrm{CDM}$ prediction at $50 \mathrm{~km} / \mathrm{s}$, concluding that the total dark matter mass in voids can at most be depleted by $20 \%$. This translates into a lower bound for the coupling of $\xi=-0.2$, see Fig. I. This is a sharp lower limit, as it relies on the results of Ref. [108], where error bars are way much smaller than the size of the effect. If we adopt a more conservative point of view, arguing that the matter is not actually missing from the voids, but that star formation in small halos is quenched, then the coupling $\xi$ will be limited to the range $-0.2<\xi<0$. This constraint is more restrictive than those obtained in Refs. [24,67]) from CMB, LSS and Supernova Ia datasets. Therefore, future, more precise estimations of matter abundance in voids could restrict the phenomenology of DEvel coupled models in a stronger way than future CMB experiments as Planck or EPIC, see Ref. [37]. As our calculations suggests, voids offer a promising avenue to the test dark coupling. To make our argument more detailed and quantitative, theoretical modeling need to be complemented by numerical simulations.

\section{Conclusions}

In this paper we have shown that, although the number of interacting cosmologies that have been proposed in the literature is vast, it is always possible to classify all existing dark coupling models in two broad families (DEvel and DMvel). Within these families we examine two subclasses of models depending on whether the coupling scales like the density of dark matter $\left(\propto \rho_{\mathrm{dm}}\right)$ or of dark energy $\left(\propto \rho_{\mathrm{de}}\right)$. At the background evolution level there are no differences between DEvel models and DMvel models: the background evolution depends only on whether the coupling scales like the density of dark matter or of dark energy. However at the perturbation level the different classes show different phenomenologies which we have explored here.

GR predicts an exact relation between $H(z)$ and the growth of structure for noninteracting dark fluids. Interactions modify this relation, and so can appear like a modification of gravity when the growth of structure and expansion history are compared precisely. We have shown that DEvel models, where there is no momentum transfer to the dark energy rest frame, induce a "fifth force" on the dark matter proportional to its peculiar velocity, violating the weak equivalence principle and deviating substantially from the uncoupled case in the growth of structure. However, for DMvel models, deviations from uncoupled growth are very small. Finally, DMvel models $\propto \rho_{\mathrm{dm}}$ are effectively indistin-

guishable from minimally coupled dark energy models with time evolving equation of state parameter. Therefore, if as a result of a forthcoming experiment, the measured expansion 
history and growth of structure are in agreement, this observation can be used to constrain deviations from GR, as commonly accepted in the literature. The conversely, however, is not necessarily true: a mis-match between expansion and growth could, indicate deviations from GR but could also, in principle indicate a dark coupling with GR unchanged. For the DMvel coupled models we considered, however, this effect is small. This is summarized in Fig. VI.

\begin{tabular}{|c|c|c|c|c|}
\hline \multicolumn{2}{|c|}{ DEvel } & \multicolumn{2}{|c|}{ DMvel } & \\
\hline \multicolumn{2}{|c|}{ GR WEPV } & \multicolumn{2}{|c|}{ GR } & \\
\hline$\propto \rho_{\mathrm{dm}}$ & $\propto \rho_{\mathrm{de}}$ & $\propto \rho_{\mathrm{dm}}$ & $\propto \rho_{\mathrm{de}}$ & \\
\hline 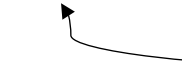 & & 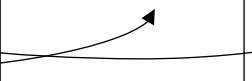 & & Identity in background \\
\hline Euler eq. & $\begin{array}{l}\text { Euler and } \\
\text { continuity eqs }\end{array}$ & background only & continuity eq. & $\begin{array}{l}\text { Modifications compared } \\
\text { to LCDM }\end{array}$ \\
\hline $\begin{array}{l}\text { Coupled } \\
\text { quintessence }\end{array}$ & $\begin{array}{r}\xi<0 \\
w_{\text {const. }}>-1 \\
\end{array}$ & $\begin{array}{r}w_{\text {const. }}<-1 \\
\text { or } w(z) \\
\end{array}$ & $\begin{array}{r}\xi<0 \\
w_{\text {const. }}>-1 \\
\end{array}$ & Ensure stability \\
\hline yes & yes & tiny & v. small & $\begin{array}{l}\text { Mis-match } \\
\text { Expansion/growth }\end{array}$ \\
\hline WEP tests & $\begin{array}{l}\text { Pec. Velocities } \\
\text { WEP tests } \\
\text { voids }\end{array}$ & $\begin{array}{c}\mathrm{H}(\mathrm{z}), \mathrm{dL}, \mathrm{Da} \\
\equiv \mathrm{w}(\mathrm{z}) \text { fluid model }\end{array}$ & $\begin{array}{l}\text { Pec. velocities } \\
\text { voids }\end{array}$ & Key observables \\
\hline$|\beta|<0.2$ & $-0.2<\xi<0$ & $-0.3<\frac{\Gamma}{H_{0}}<0.15$ & $-0.2<\xi<0$ & Current constraints \\
\hline
\end{tabular}

Figure VI: Summary of classification, phenomenology and current constraints on dark coupling models. $d_{L}$ denotes the luminosity distance as obtained from e.g. Supernovae Ia observations and $D_{a}$ denotes the angular diamater distance as measured e.g., by angular BAO.

We have analyzed how low redshift and near universe probes could be used to constrain coupled dark matter-dark energy scenarios. We have considered mis-match between highredshift (Cosmic microwave background-CMB) constraints and low redshift measurements of the background quantities such as the Hubble parameter. We have also considered tests such as the skewness, which may be promising only for DMvel \& DEvel $\propto \rho_{\text {de }}$ models; in these models deviations in the skewness for the dark matter distribution could reach the $10 \%$ level. It is however not clear whether this effect could be measured or it would be degenerate with the dark matter-galaxy bias. For the baryon distribution, (and for DMvel and DEvel $\propto \rho_{\mathrm{dm}}$ models matching CMB constraints) the deviation barely reaches $1 \%$. We have also revisited velocity-related probes, such as local bulk flows and redshift 
space distortions. Present data are not very constraining for DMvel models, but future redshift-space distortions measurements from on-going and proposed galaxy surveys have the potential to tighten significantly the allowed coupling window for both DMvel and DEvel models. Finally, we have shown that in the context of DMvel \& DEvel $\propto \rho_{\text {de }}$ models with negative coupling, the matter abundance in voids can provide a method to constrain interacting models efficiently. This test only depends on the background evolution and can be applied to any interacting model depleting or enhancing the dark matter abundance homogeneously in time.

For each of the dark coupling classes we have considered viable and stable models that fit $\mathrm{CMB}$ observations, and confronted them with present low redshift probes. We have also quantified the potential of future data. The current status on the constraints on the viable dark coupling models is summarize in Fig. VI. The reported constraints on DEvel $\propto \rho_{\mathrm{dm}}$, DEvel $\propto \rho_{\text {de }}$, DMvel $\propto \rho_{\text {dm }}$ and DMvel $\propto \rho_{\text {de }}$ come respectively from weak equivalent principle violations of galactic scales, voids, redshift-space distortions and voids.

The combination of precision tests of expansion history (BAO, $H(z)$, supernovae, matter content in voids) and growth of structure tests (peculiar velocities and weak lensing) together with weak equivalence principle tests on astronomical scales (galaxies and satellites dynamics) is the key to explore or constrain a possible coupling in the dark sector.

\section{Acknowledgments}

L. L. H was partially supported by CICYT through the project FPA2006-05423, by CAM through the project HEPHACOS, P-ESP-00346, by the PAU (Physics of the accelerating universe) Consolider Ingenio 2010, by the F.N.R.S. and the I.I.S.N.. BAR was supported by OISE/0530095. O. M. work is supported by the MICINN Ramón y Cajal contract, AYA2008-03531 and CSD2007-00060. LV is supported by FP7-PEOPLE-2007-4-3 IRG n 202182, FP7 IDEAS-Phys.LSS.240117. LV and RJ are supported by MICINN grant AYA2008-03531.

\section{A. Background evolution in models with $Q=\xi \mathcal{H} \rho_{\mathrm{de}, \mathrm{dm}}$}

For DEvel or DMvel models with $Q=\xi \mathcal{H} \rho_{\text {de }}$, the Hubble expansion rate function as a function of the redshift reads [24]

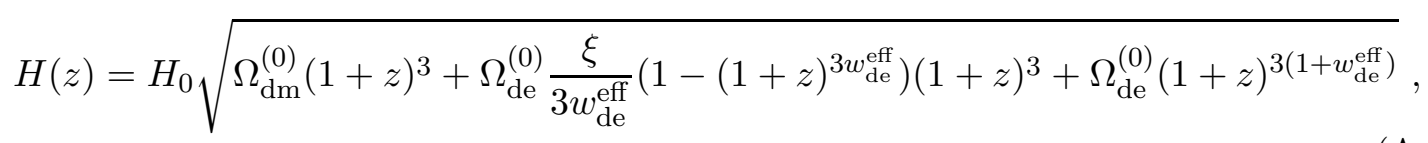

and the redshift dependent equation of state $\tilde{w}(z)$ that one would reconstruct from Eq. (3.1) reads $[24]$

$$
\tilde{w}(z)=\frac{w}{1-\frac{\xi}{3 w_{d e}^{e f f}}\left(1-(1+z)^{-3 w_{d e}^{e f f}}\right)},
$$

where $w_{\mathrm{de}}^{\mathrm{eff}}=w+\frac{\xi}{3}$. If $Q=\xi \mathcal{H} \rho_{\mathrm{dm}}$ then: 


\begin{tabular}{ccc}
\hline \hline$\xi$ & $\Omega_{\mathrm{dm}}^{0} h^{2}$ & $H_{0}(\mathrm{~km} / \mathrm{s} / \mathrm{Mpc})$ \\
\hline \hline 0 & 0.1099 & 68.29 \\
-0.1 & 0.097 & 69.48 \\
-0.2 & 0.083 & 70.68 \\
-0.3 & 0.067 & 71.90 \\
-0.4 & 0.050 & 73.13 \\
-0.5 & 0.032 & 74.37 \\
-0.6 & 0.012 & 75.63 \\
\hline \hline
\end{tabular}

Table 1: Current values of the dark matter energy density and the Hubble parameter necessaries to fit WMAP 5 year angular diameter distance data as a function of the coupling $\xi$. For numerical purposes, each time that the coupling is varied, the present day values of the cosmological parameters are choosing accordingly to the results of the fit illustrated here.

$H(z)=H_{0} \sqrt{\Omega_{\mathrm{dm}}^{(0)}(1+z)^{3\left(1+w_{\mathrm{dm}}^{\mathrm{eff}}\right)}+\Omega_{\mathrm{dm}}^{(0)} \frac{(1+z)^{3}}{1-\frac{w}{w_{\mathrm{dm}}^{\mathrm{eff}}}}\left((1+z)^{3 w}-(1+z)^{3 w_{\mathrm{dm}}^{\mathrm{eff}}}\right)+\Omega_{\mathrm{de}}^{(0)}(1+z)^{3(1+w)}}$

where $w_{\mathrm{dm}}^{\mathrm{eff}}=-\frac{\xi}{3}$. The redshift dependemt equation of state $\tilde{w}(z)$ that one would reconstruct from Eq. (3.1), reads, at small redshifts [24]

$$
\tilde{w}(z)=w\left(1+\xi \frac{\Omega_{\mathrm{dm}}^{(0)}}{\Omega_{\mathrm{de}}^{(0)}} z\right)
$$

\section{B. Cosmological parameters according to WMAP 5 constraint}

Assuming a flat universe and perfect measurements of $\Omega_{\mathrm{dm}}^{(0)} h^{2}, \Omega_{\mathrm{b}}^{(0)} h^{2}$, and the angular diameter distance to the last scattering surface from CMB observations, the amplitude of $\xi$ is degenerate with the physical energy density in dark matter today $\Omega_{\mathrm{dm}}^{(0)} h^{2}$. Table B illustrates for a selected range of couplings this effect on some of the cosmological parameters that have been used in the numerical calculations presented here.

\section{References}

[1] WMAP Collaboration, E. Komatsu et. al., Five-Year Wilkinson Microwave Anisotropy Probe (WMAP) Observations:Cosmological Interpretation, Astrophys. J. Suppl. 180 (2009) 330-376, [0803.0547].

[2] M. Kowalski et. al., Improved Cosmological Constraints from New, Old and Combined Supernova Datasets, Astrophys. J. 686 (2008) 749-778, [0804.4142].

[3] SDSS Collaboration, M. Tegmark et. al., Cosmological Constraints from the SDSS Luminous Red Galaxies, Phys. Rev. D74 (2006) 123507, [astro-ph/0608632].

[4] W. J. Percival et. al., The shape of the SDSS DR5 galaxy power spectrum, Astrophys. J. 657 (2007) 645-663, [astro-ph/0608636]. 
[5] B. A. Reid et. al., Cosmological Constraints from the Clustering of the Sloan Digital Sky Survey DR7 Luminous Red Galaxies, 0907.1659.

[6] W. J. Percival et. al., Baryon Acoustic Oscillations in the Sloan Digital Sky Survey Data Release 7 Galaxy Sample, Mon. Not. Roy. Astron. Soc. 401 (2010) 2148-2168, [0907.1660].

[7] E. Komatsu et. al., Seven-Year Wilkinson Microwave Anisotropy Probe (WMAP) Observations: Cosmological Interpretation, 1001.4538.

[8] P. J. E. Peebles and B. Ratra, Cosmology with a Time Variable Cosmological Constant, Astrophys. J. 325 (1988) L17.

[9] B. Ratra and P. J. E. Peebles, Cosmological Consequences of a Rolling Homogeneous Scalar Field, Phys. Rev. D37 (1988) 3406.

[10] C. Wetterich, The Cosmon model for an asymptotically vanishing time dependent cosmological 'constant', Astron. Astrophys. 301 (1995) 321-328, [hep-th/9408025].

[11] R. R. Caldwell, R. Dave, and P. J. Steinhardt, Quintessential cosmology: Novel models of cosmological structure formation, Astrophys. Space Sci. 261 (1998) 303-310.

[12] I. Zlatev, L.-M. Wang, and P. J. Steinhardt, Quintessence, Cosmic Coincidence, and the Cosmological Constant, Phys. Rev. Lett. 82 (1999) 896-899, [astro-ph/9807002].

[13] L.-M. Wang, R. R. Caldwell, J. P. Ostriker, and P. J. Steinhardt, Cosmic Concordance and Quintessence, Astrophys. J. 530 (2000) 17-35, [astro-ph/9901388].

[14] S. M. Carroll, Quintessence and the rest of the world, Phys. Rev. Lett. 81 (1998) 3067-3070, [astro-ph/9806099].

[15] C. J. A. P. Martins, Astrophysical Probes of Fundamental Physics, in Precision Spectroscopy in Astrophysics (N. C. Santos, L. Pasquini, A. C. M. Correia, \& M. Romaniello, ed.), pp. 89-94, 2008.

[16] L. Amendola, Coupled quintessence, Phys. Rev. D62 (2000) 043511, [astro-ph/9908023].

[17] L. Amendola, Perturbations in a coupled scalar field cosmology, Mon. Not. Roy. Astron. Soc. 312 (2000) 521, [astro-ph/9906073].

[18] L. Amendola, Scaling solutions in general non-minimal coupling theories, Phys. Rev. D60 (1999) 043501, [astro-ph/9904120].

[19] L. Amendola and D. Tocchini-Valentini, Stationary dark energy: the present universe as a global attractor, Phys. Rev. D64 (2001) 043509, [astro-ph/0011243].

[20] L. Amendola, Linear and non-linear perturbations in dark energy models, Phys. Rev. D69 (2004) 103524, [astro-ph/0311175].

[21] J. Valiviita, E. Majerotto, and R. Maartens, Instability in interacting dark energy and dark matter fluids, JCAP 0807 (2008) 020, [0804.0232].

[22] J.-H. He, B. Wang, and E. Abdalla, Stability of the curvature perturbation in dark sectors' mutual interacting models, Phys. Lett. B671 (2009) 139-145, [0807.3471].

[23] B. M. Jackson, A. Taylor, and A. Berera, On the large-scale instability in interacting dark energy and dark matter fluids, Phys. Rev. D79 (2009) 043526, [0901.3272].

[24] M. B. Gavela, D. Hernandez, L. L. Honorez, O. Mena, and S. Rigolin, Dark coupling, JCAP 0907 (2009) 034, [0901.1611]. 
[25] G. Caldera-Cabral, R. Maartens, and B. M. Schaefer, The Growth of Structure in Interacting Dark Energy Models, JCAP 0907 (2009) 027, [0905.0492].

[26] J. Valiviita, R. Maartens, and E. Majerotto, Observational constraints on an interacting dark energy model, 0907.4987.

[27] E. Majerotto, J. Valiviita, and R. Maartens, Adiabatic initial conditions for perturbations in interacting dark energy models, 0907.4981.

[28] M. B. Gavela, L. Lopez-Honorez, O. Mena, and S. Rigolin, Dark Coupling and Gauge Invariance, 1005.0295.

[29] L. Amendola, G. Camargo Campos, and R. Rosenfeld, Consequences of dark matter - dark energy interaction on cosmological parameters derived from snia data, Phys. Rev. D75 (2007) 083506, [astro-ph/0610806].

[30] B. Wang, J. Zang, C.-Y. Lin, E. Abdalla, and S. Micheletti, Interacting Dark Energy and Dark Matter: observational Constraints from Cosmological Parameters, Nucl. Phys. B778 (2007) 69-84, [astro-ph/0607126].

[31] Z.-K. Guo, N. Ohta, and S. Tsujikawa, Probing the Coupling between Dark Components of the Universe, Phys. Rev. D76 (2007) 023508, [astro-ph/0702015].

[32] G. Olivares, F. Atrio-Barandela, and D. Pavon, Dynamics of Interacting Quintessence Models: Observational Constraints, Phys. Rev. D77 (2008) 063513, [0706.3860].

[33] C. Feng, B. Wang, E. Abdalla, and R.-K. Su, Observational constraints on the dark energy and dark matter mutual coupling, Phys. Lett. B665 (2008) 111-119, [0804.0110].

[34] J.-H. He and B. Wang, Effects of the interaction between dark energy and dark matter on cosmological parameters, JCAP 0806 (2008) 010, [0801.4233].

[35] J.-H. He, B. Wang, and P. Zhang, The imprint of the interaction between dark sectors in large scale cosmic microwave background anisotropies, Phys. Rev. D80 (2009) 063530, [0906.0677].

[36] J. Bock, A. Aljabri, A. Amblard, D. Baumann, M. Betoule, T. Chui, L. Colombo, A. Cooray, D. Crumb, P. Day, C. Dickinson, D. Dowell, M. Dragovan, S. Golwala, K. Gorski, S. Hanany, W. Holmes, K. Irwin, B. Johnson, B. Keating, C. Kuo, A. Lee, A. Lange, C. Lawrence, S. Meyer, N. Miller, H. Nguyen, E. Pierpaoli, N. Ponthieu, J. Puget, J. Raab, P. Richards, C. Satter, M. Seiffert, M. Shimon, H. Tran, B. Williams, and J. Zmuidzinas, Study of the Experimental Probe of Inflationary Cosmology (EPIC)-Intemediate Mission for NASA's Einstein Inflation Probe, ArXiv e-prints (June, 2009) [0906.1188].

[37] M. Martinelli, L. L. Honorez, A. Melchiorri, and O. Mena, Future CMB cosmological constraints in a dark coupled universe, 1004.2410.

[38] K. Koyama, R. Maartens, and Y.-S. Song, Velocities as a probe of dark sector interactions, 0907.2126.

[39] O. Bertolami, F. Gil Pedro, and M. Le Delliou, Dark Energy-Dark Matter Interaction and the Violation of the Equivalence Principle from the Abell Cluster A586, Phys. Lett. B654 (2007) 165-169, [astro-ph/0703462].

[40] E. Abdalla, L. R. Abramo, L. S. Jr., and B. Wang, Signature of the interaction between dark energy and dark matter in galaxy clusters, Physics Letters B 673 (2009), no. 2107 - 110. 
[41] E. Abdalla, L. R. Abramo, and J. C. C. de Souza, Signature of the interaction between dark energy and dark matter in observations, 0910.5236.

[42] H. Kodama and M. Sasaki, Cosmological Perturbation Theory, Prog. Theor. Phys. Suppl. 78 (1984) 1-166.

[43] T. Damour, G. W. Gibbons, and C. Gundlach, Dark matter, time-varying g, and a dilaton field, Phys. Rev. Lett. 64 (Jan, 1990) 123-126.

[44] T. Damour and C. Gundlach, Nucleosynthesis constraints on an extended Jordan-BransDicke theory, Phys. Rev. D43 (1991) 3873-3877.

[45] W. Zimdahl and D. Pavon, Interacting quintessence, Phys. Lett. B521 (2001) 133-138, [astro-ph/0105479].

[46] G. R. Farrar and P. J. E. Peebles, Interacting Dark Matter and Dark Energy, Astrophys. J. 604 (2004) 1-11, [astro-ph/0307316].

[47] S. Das, P. S. Corasaniti, and J. Khoury, Super-acceleration as signature of dark sector interaction, Phys. Rev. D73 (2006) 083509, [astro-ph/0510628].

[48] H.-S. Zhang and Z.-H. Zhu, Interacting Chaplygin gas, Phys. Rev. D73 (2006) 043518, [astro-ph/0509895].

[49] S. del Campo, R. Herrera, G. Olivares, and D. Pavon, Interacting models of soft coincidence, Phys. Rev. D74 (2006) 023501, [astro-ph/0606520].

[50] R. Bean, E. E. Flanagan, and M. Trodden, The Adiabatic Instability on Cosmology's Dark Side, New J. Phys. 10 (2008) 033006, [0709.1124].

[51] C. G. Boehmer, G. Caldera-Cabral, N. Chan, R. Lazkoz, and R. Maartens, Quintessence with quadratic coupling to dark matter, Phys. Rev. D81 (2010) 083003, [0911.3089].

[52] C. Brans and R. H. Dicke, Mach's Principle and a Relativistic Theory of Gravitation, Physical Review 124 (Nov., 1961) 925-935.

[53] T. P. Sotiriou and V. Faraoni, f(R) Theories Of Gravity, Rev. Mod. Phys. 82 (2010) 451-497, [0805.1726].

[54] A. De Felice and S. Tsujikawa, $f(R)$ theories, Living Rev. Rel. 13 (2010) 3, [1002.4928].

[55] L. Amendola and D. Tocchini-Valentini, Baryon bias and structure formation in an accelerating universe, PRD 66 (Aug., 2002) 043528-+, [arXiv:astro-ph/0111535].

[56] M. Ishak, A. Upadhye, and D. N. Spergel, Probing cosmic acceleration beyond the equation of state: Distinguishing between dark energy and modified gravity models, PRD $\mathbf{7 4}$ (Aug., 2006) 043513-+, [arXiv:astro-ph/0507184].

[57] V. Acquaviva and L. Verde, Observational signatures of Jordan Brans Dicke theories of gravity, Journal of Cosmology and Astro-Particle Physics 12 (Dec., 2007) 1-+, [0709.0082].

[58] B. Jain and P. Zhang, Observational tests of modified gravity, PRD 78 (Sept., 2008) 063503-+, [0709.2375].

[59] Y. Song and W. J. Percival, Reconstructing the history of structure formation using redshift distortions, Journal of Cosmology and Astro-Particle Physics 10 (Oct., 2009) 4-+, [0807.0810]. 
[60] L. Guzzo et. al., A test of the nature of cosmic acceleration using galaxy redshift distortions, Nature 451 (2008) 541-545, [0802 .1944].

[61] F. Simpson, B. M. Jackson, and J. A. Peacock, Unmodified Gravity, 1004.1920.

[62] G. Ballesteros and J. Lesgourgues, Dark energy with non-adiabatic sound speed: initial conditions and detectability, 1004.5509.

[63] S. Chongchitnan, Cosmological Perturbations in Models of Coupled Dark Energy, Phys. Rev. D79 (2009) 043522, [0810.5411].

[64] P. S. Corasaniti, Slow-Roll Suppression of Adiabatic Instabilities in Coupled Scalar Field-Dark Matter Models, Phys. Rev. D78 (2008) 083538, [0808.1646].

[65] R. Bean, E. E. Flanagan, and M. Trodden, Adiabatic instability in coupled dark energy-dark matter models, Phys. Rev. D78 (2008) 023009, [0709.1128].

[66] C. Clarkson, M. Cortes, and B. A. Bassett, Dynamical dark energy or simply cosmic curvature?, JCAP 0708 (2007) 011, [astro-ph/0702670].

[67] L. L. Honorez and. Mena, Instabilities in dark coupled models and constraints from cosmological data, 0911.3269.

[68] A. Lewis, A. Challinor, and A. Lasenby, Efficient computation of CMB anisotropies in closed FRW models, Astrophys. J. 538 (2000) 473-476, [astro-ph/9911177].

[69] A. Lewis and S. Bridle, Cosmological parameters from CMB and other data: a Monte- Carlo approach, Phys. Rev. D66 (2002) 103511, [astro-ph/0205436].

[70] A. F. Heavens, T. D. Kitching, and L. Verde, On model selection forecasting, Dark Energy and modified gravity, Mon. Not. Roy. Astron. Soc. 380 (2007) 1029-1035, [astro-ph/0703191].

[71] L. Verde, H. Peiris, and R. Jimenez, Optimizing CMB polarization experiments to constrain inflationary physics, JCAP 0601 (2006) 019, [astro-ph/0506036].

[72] W. L. Freedman and B. F. Madore, The Hubble Constant, 1004.1856.

[73] L. Amendola and C. Quercellini, Skewness as a test of the equivalence principle, Phys. Rev. Lett. 92 (2004) 181102, [astro-ph/0403019].

[74] M. Kamionkowski and A. Buchalter, Weakly Nonlinear Clustering for Arbitrary Expansion Histories, Astrophys. J. 514 (1999) 7, [astro-ph/9807211].

[75] F. R. Bouchet, S. Colombi, E. Hivon, and R. Juszkiewicz, Perturbative Lagrangian approach to gravitational instability., A $6 A 296$ (Apr., 1995) 575-+, [arXiv:astro-ph/9406013].

[76] P. Catelan, F. Lucchin, S. Matarrese, and L. Moscardini, Eulerian perturbation theory in non-flat universes: second-order approximation, MNRAS 276 (Sept., 1995) 39-56, [arXiv: astro-ph/9411066].

[77] R. Watkins, H. A. Feldman, and M. J. Hudson, Consistently Large Cosmic Flows on Scales of $100 \mathrm{Mpc} / \mathrm{h}$ : a Challenge for the Standard LCDM Cosmology, 0809.4041.

[78] Y. Ayaita, M. Weber, and C. Wetterich, Peculiar Velocity Anomaly from Forces Beyond Gravity?, 0908.2903.

[79] A. V. Maccio, C. Quercellini, R. Mainini, L. Amendola, and S. A. Bonometto, N-body simulations for coupled dark energy: halo mass function and density profiles, Phys. Rev. D69 (2004) 123516, [astro-ph/0309671]. 
[80] M. Baldi, V. Pettorino, G. Robbers, and V. Springel, Hydrodynamical N-body simulations of coupled dark energy cosmologies, MNRAS 403 (Apr., 2010) 1684-1702.

[81] J. Dunlop, J. Peacock, H. Spinrad, A. Dey, R. Jimenez, D. Stern, and R. Windhorst, A 3.5-Gyr-old galaxy at redshift 1.55, Nature $\mathbf{3 8 1}$ (June, 1996) 581-584.

[82] H. Spinrad, A. Dey, D. Stern, J. Dunlop, J. Peacock, R. Jimenez, and R. Windhorst, LBDS 53W091: an Old, Red Galaxy at z=1.552, ApJ 484 (July, 1997) 581-+, [arXiv:astro-ph/9702233].

[83] L. L. Cowie, A. Songaila, and A. J. Barger, Evidence for a Gradual Decline in the Universal Rest-Frame Ultraviolet Luminosity Density for Z i 1, A.J. 118 (Aug., 1999) 603-612, [arXiv:astro-ph/9904345].

[84] A. Heavens, B. Panter, R. Jimenez, and J. Dunlop, The star-formation history of the Universe from the stellar populations of nearby galaxies, arXiv:astro-ph/0403293.

[85] D. Thomas, C. Maraston, R. Bender, and C. Mendes de Oliveira, The Epochs of Early-Type Galaxy Formation as a Function of Environment, arXiv: astro-ph/0410209.

[86] B. Panter, R. Jimenez, A. F. Heavens, and S. Charlot, The star formation histories of galaxies in the Sloan Digital Sky Survey, Mon. Not. Roy. Astron. Soc. 378 (2007) 1550-1564, [astro-ph/0608531].

[87] R. Jimenez, B. Panter, A. F. Heavens, and L. Verde, Baryonic conversion tree: the global assembly of stars and dark matter in galaxies from the Sloan Digital Sky Survey, arXiv: astro-ph/0403294.

[88] T. Treu, R. S. Ellis, T. X. Liao, P. G. van Dokkum, P. Tozzi, A. Coil, J. Newman, M. C. Cooper, and M. Davis, The Assembly History of Field Spheroidals: Evolution of Mass-to-Light Ratios and Signatures of Recent Star Formation, arXiv:astro-ph/0503164.

[89] R. Mandelbaum, U. Seljak, G. Kauffmann, C. M. Hirata, and J. Brinkmann, Galaxy halo masses and satellite fractions from galaxy-galaxy lensing in the Sloan Digital Sky Survey: stellar mass, luminosity, morphology and environment dependencies, MNRAS 368 (May, 2006) 715-731, [arXiv:astro-ph/0511164].

[90] N. Kaiser, Clustering in real space and in redshift space, Mon. Not. Roy. Astron. Soc. 227 (1987) 1-27.

[91] Y.-S. Song and W. J. Percival, Reconstructing the History of Structure Formation using Peculiar Velocities, 0807.0810.

[92] M. White, Y.-S. Song, and W. J. Percival, Forecasting Cosmological Constraints from Redshift Surveys, 0810.1518.

[93] M. Kesden and M. Kamionkowski, Galilean Equivalence for Galactic Dark Matter, Phys. Rev. Lett. 97 (2006) 131303, [astro-ph/0606566].

[94] M. Kesden and M. Kamionkowski, Tidal Tails Test the Equivalence Principle in the Dark Sector, Phys. Rev. D74 (2006) 083007, [astro-ph/0608095].

[95] L. Amendola, Coupled quintessence, PRD 62 (Aug., 2000) 043511-+, [arXiv:astro-ph/9908023].

[96] J. A. Frieman and B. Gradwohl, Dark matter and the equivalence principle, Physical Review Letters 67 (Nov., 1991) 2926-2929. 
[97] C. Wetterich, An asymptotically vanishing time-dependent cosmological "constant"., A\&A 301 (Sept., 1995) 321-+, [arXiv:hep-th/9408025].

[98] R. Bean, E. E. Flanagan, I. Laszlo, and M. Trodden, Constraining Interactions in Cosmology's Dark Sector, Phys. Rev. D78 (2008) 123514, [0808.1105].

[99] T. Koivisto, Growth of perturbations in dark matter coupled with quintessence, Phys. Rev. D72 (2005) 043516, [astro-ph/0504571].

[100] L. Amendola and C. Quercellini, Tracking and coupled dark energy as seen by WMAP, Phys. Rev. D68 (2003) 023514, [astro-ph/0303228].

[101] J. A. Keselman, A. Nusser, and P. J. E. Peebles, Galaxy Satellites and the Weak Equivalence Principle, Phys. Rev. D80 (2009) 063517, [0902.3452].

[102] J. A. Keselman, A. Nusser, and P. J. E. Peebles, Cosmology with Equivalence Principle Breaking in the Dark Sector, 0912.4177.

[103] C. Sealfon, L. Verde, and R. Jimenez, Limits on deviations from the inverse-square law on megaparsec scales, PRD 71 (Apr., 2005) 083004-+, [arXiv:astro-ph/0404111].

[104] O. Doré, M. Martig, Y. Mellier, M. Kilbinger, J. Benjamin, L. Fu, H. Hoekstra, M. Schultheis, E. Semboloni, and I. Tereno, Testing Gravity with the CFHTLS-Wide Cosmic Shear Survey and SDSS LRGs, ArXiv e-prints (Dec., 2007) [0712.1599].

[105] J.-Q. Xia, Constraint on coupled dark energy models from observations, Phys. Rev. D80 (2009) 103514, [0911.4820].

[106] M. Baldi, Time dependent couplings in the dark sector: from background evolution to nonlinear structure formation, 1005.2188.

[107] P. J. E. Peebles, The Void Phenomenon, Astrophys. J. 557 (2001) 495-504, [astro-ph/0101127].

[108] A. Tikhonov and A. Klypin, The emptiness of voids: yet another over-abundance problem for the LCDM model, 0807.0924.

[109] J. Binney and M. Merrifield, Galactic astronomy. 1998.

[110] R. Jimenez, A. F. Heavens, M. R. S. Hawkins, and P. Padoan, Dark galaxies, spin bias and gravitational lenses, astro-ph/9709050.

[111] L. Verde, S. P. Oh, and R. Jimenez, The abundance of dark galaxies, Mon. Not. Roy. Astron. Soc. 336 (2002) 541, [astro-ph/0202283].

[112] S. Trujillo-Gomez, A. Klypin, J. Primack, and A. J. Romanowsky, LCDM Correctly Predicts Basic Statistics of Galaxies: Luminosity-Velocity Relation, Baryonic Mass-Velocity Relation, and Velocity Function, ArXiv e-prints (May, 2010) [1005.1289].

[113] A. Ferrara and E. Tolstoy, The role of stellar feedback and dark matter in the evolution of dwarf galaxies, MNRAS 313 (Apr., 2000) 291-309, [arXiv:astro-ph/9905280]. 